\title{
Posture control of a 3-RPS pneumatic parallel platform with parameter initialization and an adaptive robust method ${ }^{*}$
}

\author{
Guo-liang TAO ${ }^{\ddagger 1}$, Ce SHANG ${ }^{1}$, De-yuan $\mathrm{MENG}^{2}$, Chao-chao ZHOU ${ }^{1}$ \\ $\left({ }^{1}\right.$ The State Key Laboratory of Fluid Power Transmission and Control, Zhejiang University, Hangzhou 310027, China) \\ ( ${ }^{2}$ School of Mechatronic Engineering, China University of Mining and Technology, Xuzhou 221116, China) \\ E-mail: gltao@zju.edu.cn; czesh@zju.edu.cn; mengdeyuan8207@163.com; 21425044@zju.edu.cn \\ Received Oct. 21, 2015; Revision accepted Mar. 21, 2016; Crosschecked Feb. 28, 2017
}

\begin{abstract}
A control algorithm for a 3-RPS parallel platform driven by pneumatic cylinders is discussed. All cylinders are controlled by proportional directional valves while the kinematic and dynamic properties of the system are modeled. The method of adaptive robust control is applied to the controller using a back-stepping approach and online parameter estimation. To compensate for the uncertainty and the influence caused by estimations, a fast dynamic compensator is integrated in the controller design. To prevent any influence caused by the load applied to the moving platform changing in a practical working situation, the identification of parameters is taken as the initialization of unknown parameters in the controller, which can improve the adaptability of the algorithm. Using these methods, the response rate of the parameter estimation and control performance were improved significantly. The adverse effects of load and restriction forces were eliminated by the initialization and online estimation. Experiments under different situations illustrated the effectiveness of the adaptive robust controller with parameter initialization, approaching average tracking errors of less than $1 \%$.
\end{abstract}

Key words: Parameter initialization; Adaptive robust control; Parallel mechanism; Pneumatic cylinders http://dx.doi.org/10.1631/FITEE.1500353

CLC number: TP273

\section{Introduction}

Parallel mechanisms are now used widely in many industrial and civil applications, such as motion simulators, adjustment platforms, and virtual reality equipment (Merlet, 2002). Compared with a full six-degree-of-freedom (6-DoF) structure, such as a Stewart platform, the incomplete DoF 3-RPS (parallel mechanism that has three chains with revolute $(\mathrm{R})$, prismatic $(\mathrm{P})$, and spherical $(\mathrm{S})$ joints in each one) manipulator retains the three most important

\footnotetext{
$\ddagger$ Corresponding author

* Project supported by the National Natural Science Foundation of China (No. 51375430)

(D) ORCID: Guo-liang TAO, http://orcid.org/0000-0003-1643-6047 (C)Zhejiang University and Springer-Verlag Berlin Heidelberg 2017
}

freedoms - roll, pitch, and heave - of a virtual reality entertainment moving stage. As a type of parallel mechanism, it has the advantages of heavy load capacity, high rigidity, and low inertia (Pfreundschuh et al., 1991). Because there are only three actuators driving the moving platform, the hardware costs can be lower and the controller structure can be simpler. Many mechanism and structural analyses have been conducted regarding its kinematic and dynamic properties (Wang et al., 2005; Zheng et al., 2011). Researchers have also studied realistic devices that have 3-RPS structures and similar types. To control the posture of the platform, the accuracy of the model parameters is the key to performance control. Because there are seven moving parts and friction 
forces exist in all joints, all the inertial, frictional, and load parameters will lead to a large observation matrix and parameter vector. Farhat and DíazRodríguez proposed methods for this kind of identification process (Farhat et al., 2008; Díaz-Rodríguez et al., 2010).

Many 3-RPS platforms that are used currently are driven by electric or hydraulic cylinders to provide adequate control precision. However, for some occasions that have the large demands of 3-RPS platforms, the lower cost advantage of pneumatic cylinders makes them part of the market, even though the trajectory tracking accuracy is much lower than those of electric and hydraulic devices. Due to the compressibility of air and nonlinear characteristics of pneumatic elements, pneumatic servo control is also much more difficult. Improvements in pneumatic servo control performance have long been a goal of researchers and engineers. During recent years, many studies had been performed in this area with many algorithms applied to pneumatic systems, such as feedback linearization, self-tuning, fuzzy, and sliding mode controls (Kimura et al., 1997; Richardson et al., 2001; Schulte and Hahn, 2004; Girin et al., 2009). Based on the back-stepping method, the adaptive robust controller has attracted researchers' attention for its resistance to parameter uncertainties and nonlinear characteristics (Yao and Tomizuka, 1997; Smaoui et al., 2006). In recent years, the developed theories have been successfully applied in mechatronic control systems based on linear motors (Chen et al., 2013a; 2013b) and hydraulic elements (Yao et al., 2014b), in addition to theoretical improvements (Yao et al., 2014a; Chen et al., 2015). Applying adaptive robust method aspects in the controller design has been shown to be an effective way to improve the pneumatic servo system position control performance for a single cylinder (Meng et al., 2013).

With developments in parallel mechanism analysis, pneumatic modeling, and control methods, research advances in posture tracking control for a parallel platform driven by pneumatic elements have been reported recently. For the parallel platform used in virtual reality entertainment equipment (a four-dimensional (4D) movie chair), posture condition and load forces vary heavily during ordinary operation. This is quite different from the singlecylinder experiments in the studies mentioned above. To eliminate this defect, one choice is the use of pneu- matic muscles, in which frictional forces do not exist and their influences would not appear (Zhu et al., 2008; 2009; Tao and Zuo, 2014). However, the pneumatic muscle can bear only one-directional tensile force, which means it cannot be used in heavy load conditions under compression forces. For a manipulator driven by cylinders, which can accept forces from both sides, some work has also been done with different control methods (Grewal et al., 2011; 2012; Ramsauer et al., 2012; Pradipta et al., 2013; Andrievsky et al., 2014). The precisions of the platforms driven by pneumatic cylinders in these studies were about 5\%-20\% and load-sensitive. An entertainment $4 \mathrm{D}$ movie platform should bear about a 200-kg load or more; the dynamic model would change as the load varies in a realistic working situation. This may lead to controller instability without a robust controller. Moreover, in the application of multiple parallel platforms, the parameters assumed to be constant in value during controller design may differ in different manufacturing batches, such as the dead-zone and friction properties.

In this study, based on aspects of adaptive robust control, a controller is designed accordingly for a pneumatic parallel system model. Stability is demonstrated numerically and the effectiveness is validated through simulations and experiments. Compared with the basic adaptive robust method, a direct part is integrated into the robust controller design to raise the transient performance. Moreover, a new method integrated with parameter initialization (ARCPI) is proposed for posture control for the parallel pneumatic manipulator to overcome the disadvantages caused by the variation of the initial estimation value. The adaptability of this control algorithm satisfies realistic working situations, and the controller has much better tracking performance. In the second section, the kinematic and dynamic models of the 3-RPS platform are established with mechanical constraints. Online load estimation, based on a least-squares method and noncontinuous projection mapping, is proposed in Section 3.1. The inertial and frictional parameters can be initialized while using identification technology to resist load changes and time-varying features, as discussed in Section 3.2. A fast dynamic compensator is introduced into the robust controller design, based on the dynamic model of the 3-RPS system, as described in Section 3.3. Based on the modeling and controller 
design, Section 4 provides the experimental results, confirming the effectiveness of ARC and indicating that the improved algorithm (ARCPI) can make the pneumatic platform resist the effects of load variation and strengthen the adaptability of the controller. A summary and discussion are provided in the final section. Proof of stability of the controller is provided in the Appendix.

\section{Modeling of a 3-RPS pneumatic plat- form}

\subsection{Kinematics}

The transition matrix is shown in the form of Tait-Bryan angles in Eq. (1), where $s$ represents sin and $c$ represents cos. $\left({ }^{A} X_{\mathrm{bo}},{ }^{A} Y_{\mathrm{bo}},{ }^{A} Z_{\mathrm{bo}}\right)$ is the location of the origin of the moving board in the fixed board coordinate:

$$
\begin{aligned}
& { }_{B}^{A} R= \\
& {\left[\begin{array}{ccc}
c \beta c \gamma & -c \beta s \gamma & s \beta \\
c \alpha s \gamma+s \alpha s \beta c \gamma & c \alpha c \gamma-s \alpha s \beta s \gamma & -s \alpha c \beta \\
s \alpha s \gamma-c \alpha s \beta c \gamma & c \gamma s \alpha+c \alpha s \beta s \gamma & c \alpha c \beta
\end{array}\right],}
\end{aligned}
$$

where $r_{A}$ and $r_{B}$ are the lengths of $O_{A} A_{i}$ and $O_{B} B_{i}$, respectively. In the current structure, the joint points $A_{i}$ and $B_{i}$ all locate on the same radius circle on each plane, and $O_{A}$ and $O_{B}$ are the centroids of the equilateral triangles $A_{1} A_{2} A_{3}$ and $B_{1} B_{2} B_{3}$, respectively. Points $A_{i}, B_{i}, O_{A}, O_{B}$ are in the same plane. Fig. 1 shows the mechanical structure and the coordinate configurations. $\boldsymbol{u}_{i}$ is the normalized vector of the rotational joint axis. $\boldsymbol{n}_{i}$ is the normalized vector of $\boldsymbol{L}_{i}$.

According to the mechanical structure, the constraint equations can be written as

$$
\left\{\begin{array}{l}
{ }^{A} Y_{\mathrm{bo}}=r_{B}(\cos \beta \sin \gamma) \\
{ }^{A} X_{\mathrm{bo}}=\frac{r_{B}}{2}(\cos \beta \cos \gamma \\
\quad+\sin \alpha \sin \beta \sin \gamma-\cos \alpha \cos \gamma) \\
\gamma=-\arctan \left(\frac{\sin \alpha \sin \beta}{\cos \alpha+\cos \beta}\right)
\end{array}\right.
$$

When the desired trajectory $\boldsymbol{X}_{\mathrm{d}}$ in working space $\{\alpha(t), \beta(t), Z(t)\}$ is given, according to the coordinate definition in Fig. 1 and the constraints in Eq. (2), the lengths of all actuators can be calcu- lated in vector form:

$$
\begin{aligned}
\boldsymbol{L}_{i} & ={ }_{B}^{A} T^{B} \boldsymbol{P}_{\mathrm{s} i}-{ }^{A} \boldsymbol{P}_{\mathrm{R} i} \\
& ={ }_{B}^{A} R_{x y z}{ }^{B} \boldsymbol{P}_{\mathrm{s} i}+{ }^{A} \boldsymbol{P}_{\mathrm{bo}}-{ }^{A} \boldsymbol{P}_{\mathrm{R} i},
\end{aligned}
$$

where $\boldsymbol{L}_{i}$ is the vector of each cylinder piston rod's direction in the fixed coordinate. $l_{i}=\left|\boldsymbol{L}_{i}\right|-L_{0 i}, L_{0 i}$ is the original length of cylinder $i$, and $l_{i}$ is the actual position of each piston rod. Thus, the transition functions from workspace $\boldsymbol{X}_{\mathrm{d}}(\alpha, \beta, z)$ to joint space $\boldsymbol{x}_{\mathrm{d}}\left(l_{1}, l_{2}, l_{3}\right)$ are established. Fig. 2 is the physical platform connected to the signal conditioning board (PCB) and NI-PXI controller.
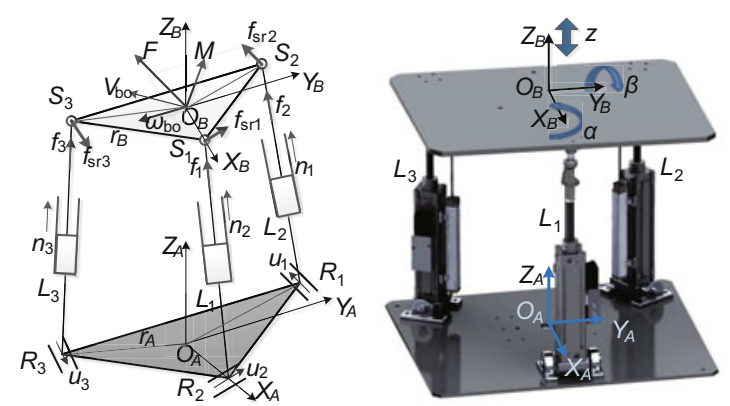

Fig. 1 Mechanical structure of the 3-RPS pneumatic platform

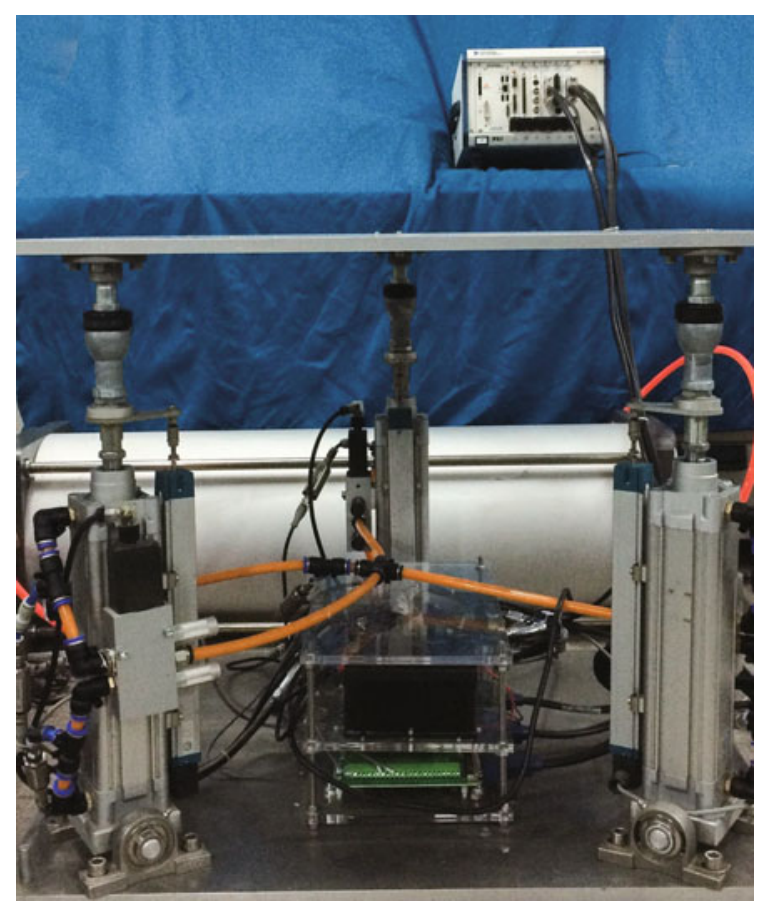

Fig. 2 Physical platform 


\subsection{Dynamics}

According to Carneiro and de Almeida (2007) and Meng et al. (2011), a polytropic process is chosen as a more accurate description of the thermodynamic process in cylinder chambers:

$$
\left\{\begin{aligned}
& T_{i}= T_{\mathrm{s}}\left(\frac{p_{i}}{p_{\mathrm{bal}}}\right)^{\frac{n-1}{n}}, p_{\mathrm{bal}}=0.8077 p_{\mathrm{s}}, \\
& \frac{\mathrm{d} p_{i}}{\mathrm{~d} t}= \frac{\gamma_{\mathrm{a}} R}{V_{i}}\left(\dot{m}_{i \mathrm{in}} T_{\mathrm{s}}-\dot{m}_{i \mathrm{out}} T_{i}\right) \\
& \quad \quad-\frac{\gamma_{\mathrm{a}} p_{i}}{V_{i}} \frac{\mathrm{d} V_{i}}{\mathrm{~d} t}+\frac{\gamma_{\mathrm{a}}-1}{V_{i}} \dot{Q}_{i}+d_{i n}+\tilde{d}_{i 0}, \quad i=a, b \\
& V_{i}= V_{0 i}+A_{i}(L / 2+x), \\
& \dot{Q}_{i}=h S_{\mathrm{h} i}(x)\left(T_{\mathrm{s}}-T_{i}\right) \\
& S_{\mathrm{h} i}(x)=2 A_{i}+\pi D(L / 2+x),
\end{aligned}\right.
$$

where $h$ is the thermal conductivity of air and the inner chamber surface, $S_{\mathrm{h} i}(x)$ is the area of heat exchange, $p_{\mathrm{s}}$ is the source pressure, $\gamma_{\mathrm{a}}$ is the heat capacity ratio, and $R$ is the ideal gas constant. Because the natural frequency of the proportional directional control valve (FESTO MPYE series) is much higher than the bandwidth of the pneumatic servo system, the dynamic feature of the valve core can be ignored. The valve model in Eq. (5) is used to calculate the mass flow:

$$
\begin{aligned}
& q_{\mathrm{m}}= \begin{cases}A(u) C_{d} C_{a} P_{\mathrm{u}} / \sqrt{T_{\mathrm{u}}}, & P_{\mathrm{d}} / P_{\mathrm{u}}<p_{\mathrm{r}}, \\
A(u) C_{d} C_{a} C_{b} P_{\mathrm{u}} / \sqrt{T_{\mathrm{u}}}, & p_{\mathrm{r}}<P_{\mathrm{d}} / P_{\mathrm{u}}<\lambda, \\
A(u) C_{d} C_{a} C_{c} P_{\mathrm{u}} / \sqrt{T_{\mathrm{u}}}, & \lambda<P_{\mathrm{d}} / P_{\mathrm{u}}<1,\end{cases} \\
& \left\{\begin{array}{l}
C_{a}=\sqrt{\left(\gamma_{\mathrm{a}} / R\right)\left(2 /\left(\gamma_{\mathrm{a}}+1\right)\right)^{\left(\gamma_{\mathrm{a}}+1\right) /\left(\gamma_{\mathrm{a}}-1\right)},} \\
C_{b}=\sqrt{1-\left(P_{\mathrm{d}} / P_{\mathrm{u}}-p_{\mathrm{r}}\right)^{2} /\left(1-p_{\mathrm{r}}\right)^{2}}, \\
C_{c}=\frac{P_{\mathrm{d}} / P_{\mathrm{u}}-p_{\mathrm{r}}}{1-\lambda} \sqrt{1-\left(\frac{\lambda-p_{\mathrm{r}}}{1-p_{\mathrm{r}}}\right)^{2}}, \\
C_{d}=1.099-0.1075 P_{\mathrm{d}} / P_{\mathrm{u}},
\end{array}\right.
\end{aligned}
$$

where $P_{\mathrm{d}}$ and $P_{\mathrm{u}}$ are the downstream and upstream pressures, respectively, $p_{\mathrm{r}}$ is the critical pressure ratio, and $\lambda$ is the linear flow ratio.

Based on the structure of the 3-RPS pneumatic platform, the dynamic model in the joint space can be written as follows:

$$
\left\{\begin{array}{l}
\boldsymbol{M}_{\mathrm{e}} \ddot{\boldsymbol{x}}=\overline{A_{\mathrm{a}}} \boldsymbol{p}_{\mathrm{L}}-\boldsymbol{b} \dot{\boldsymbol{x}}-\boldsymbol{A}_{\mathrm{f}} \boldsymbol{S}_{\mathrm{f}}(\dot{\boldsymbol{x}})-\boldsymbol{F}_{\mathrm{L}}+\boldsymbol{f}_{\mathrm{n}}+\widetilde{\boldsymbol{f}}_{0} \\
\dot{\boldsymbol{p}}_{\mathrm{L}}=\boldsymbol{q}_{\mathrm{L}}+\boldsymbol{F}_{\mathrm{p}}+\boldsymbol{d}_{\mathrm{n}}+\widetilde{\boldsymbol{d}}_{0}
\end{array}\right.
$$

where $\boldsymbol{M}_{\mathrm{e}}=\operatorname{diag}\left(m_{1}, m_{2}, m_{3}\right), m_{i}$ stands for the equivalent inertial load of each piston $\operatorname{rod}, \bar{A}_{\mathrm{a}}=$
$H_{\mathrm{p}} A_{\mathrm{a}} \cdot \boldsymbol{x}=\left[x_{1}, x_{2}, x_{3}\right]^{\mathrm{T}}$ is the desired position curve of each cylinder, $\boldsymbol{p}_{\mathrm{L}}=\left[p_{\mathrm{L} 1}, p_{\mathrm{L} 2}, p_{\mathrm{L} 3}\right]^{\mathrm{T}}, p_{\mathrm{L} i}=p_{i a}-$ $k_{i \text { area }} p_{i b}$, and $k_{i \text { area }}$ is the area factor for the cylinder chambers within the piston rod. $\boldsymbol{p}_{\mathrm{L}}$ is the first-level virtual input. The physical meaning of $p_{\mathrm{L} i}$ is the equivalent pressure difference between the two chambers of one cylinder. $\quad \boldsymbol{q}_{\mathrm{L}}=\left[q_{\mathrm{L} 1}, q_{\mathrm{L} 2}, q_{\mathrm{L} 3}\right]^{\mathrm{T}}$, which stands for the second-level virtual input, is used as the desired flow rate to calculate the control voltages of the proportional valves. $\boldsymbol{F}_{\mathrm{p}}=\left[F_{\mathrm{p} 1}, F_{\mathrm{p} 2}, F_{\mathrm{p} 3}\right]^{\mathrm{T}}$ is the load matrix applied to cylinder piston rods. $\tilde{\boldsymbol{f}}_{\mathrm{n}}=\left[\tilde{f}_{\mathrm{n} 1}, \tilde{f}_{\mathrm{n} 2}, \tilde{f}_{\mathrm{n} 3}\right]^{\mathrm{T}}$ and $\tilde{\boldsymbol{f}}_{\mathrm{o}}=\left[\tilde{f}_{\mathrm{o} 1}, \tilde{f}_{\mathrm{o} 2}, \tilde{f}_{\mathrm{o} 3}\right]^{\mathrm{T}}$ represent the modeling error and disturbance on each axis, respectively. $\boldsymbol{d}_{\mathrm{n}}=\left[d_{\mathrm{n} 1}, d_{\mathrm{n} 2}, d_{\mathrm{n} 3}\right]^{\mathrm{T}}$ and $\tilde{\boldsymbol{d}}_{\mathrm{o}}=$ $\left[d_{\mathrm{o} 1}, d_{\mathrm{o} 2}, d_{\mathrm{o} 3}\right]^{\mathrm{T}}$ are the influence caused by the valve model and disturbance of air flow, respectively. $\tilde{\boldsymbol{f}}_{\mathrm{o}}$ and $\tilde{\boldsymbol{d}}_{\mathrm{o}}$ are the nonlinear uncertainty parts in the model. $H_{\mathrm{p}}$ is constant at $10^{5}$.

$$
\left\{\begin{aligned}
q_{\mathrm{L} i}= & \frac{\gamma_{\mathrm{a}} R}{H_{\mathrm{p}} V_{i a}}\left(\dot{m}_{i a \mathrm{in}} T_{\mathrm{s}}-\dot{m}_{i a \mathrm{out}} T_{i a}\right) \\
& -\frac{\gamma_{\mathrm{a}} R}{H_{\mathrm{p}} V_{i b}}\left(\dot{m}_{i b i n} T_{\mathrm{s}}-\dot{m}_{i b \text { out }} T_{i b}\right), \\
F_{\mathrm{p} i}= & -\frac{\gamma_{\mathrm{a}} A_{i a}}{V_{i a}} \dot{x}_{i} p_{i a}-\frac{\gamma_{\mathrm{a}} A_{i b}}{V_{i b}} \dot{x}_{i} p_{i b} \\
& +\frac{\gamma_{\mathrm{a}}-1}{H_{\mathrm{p}} V_{i a}} \dot{Q}_{i a}-\frac{\gamma_{\mathrm{a}}-1}{H_{\mathrm{p}} V_{i b}} \dot{Q}_{i b} .
\end{aligned}\right.
$$

Eq. (7) gives the mathematical expression of $q_{\mathrm{L} i}$ and $F_{\mathrm{L} i}$. Next, we define the unknown parameter vectors $\boldsymbol{\theta}_{a}, \boldsymbol{\theta}_{b}$ and their regression matrices $\boldsymbol{\varphi}_{a}, \boldsymbol{\varphi}_{b}$ as shown in Eqs. (8)-(10):

$$
\begin{aligned}
& \left\{\begin{array}{l}
\boldsymbol{\theta}_{a}=\left[\theta_{1}, \theta_{2}, \theta_{3}\right]^{\mathrm{T}}, \\
\boldsymbol{\theta}_{i}=\left[b_{i}, A_{\mathrm{f} i},-F_{\mathrm{L} i}+f_{\mathrm{n} i}\right]^{\mathrm{T}}, i=1,2,3, \\
\boldsymbol{\theta}_{b}=\left[\theta_{b 1}, \theta_{b 2}, \theta_{b 3}\right]^{\mathrm{T}}=\left[d_{\mathrm{n} 1}, d_{\mathrm{n} 2}, d_{\mathrm{n} 3}\right]^{\mathrm{T}},
\end{array}\right. \\
& \boldsymbol{\varphi}_{a}=\left[\begin{array}{ccc}
\dot{x}_{1} & 0 & 0 \\
S_{\mathrm{f} 1}\left(\dot{x}_{1}\right) & 0 & 0 \\
1 & 0 & 0 \\
0 & \dot{x}_{2} & 0 \\
0 & S_{\mathrm{f} 2}\left(\dot{x}_{2}\right) & 0 \\
0 & 1 & 0 \\
0 & 0 & \dot{x}_{3} \\
0 & 0 & S_{\mathrm{f} 3}\left(\dot{x}_{3}\right) \\
0 & 0 & 1
\end{array}\right], \\
& \boldsymbol{\varphi}_{b}^{\mathrm{T}}=\boldsymbol{I}_{3 \times 3} .
\end{aligned}
$$

Finally, the simplified dynamic model of the upper board driven by three pneumatic cylinders under proportional valve control is established in the following equations:

$$
\left\{\begin{array}{l}
\boldsymbol{M}_{\mathrm{e}} \ddot{\boldsymbol{x}}=\overline{A_{\mathrm{a}}} \boldsymbol{p}_{\mathrm{L}}+\boldsymbol{\varphi}_{a}^{\mathrm{T}} \boldsymbol{\theta}_{a}+\widetilde{\boldsymbol{f}}_{0}, \\
\dot{\boldsymbol{p}}_{\mathrm{L}}=\boldsymbol{q}_{\mathrm{L}}+\boldsymbol{F}_{\mathrm{p}}+\boldsymbol{\varphi}_{b}^{\mathrm{T}} \boldsymbol{\theta}_{b}+\widetilde{\boldsymbol{d}}_{0}
\end{array}\right.
$$




$$
\left\{\begin{array}{l}
\boldsymbol{\theta}_{i} \in \boldsymbol{B}_{\boldsymbol{\theta}_{i}} \\
\tilde{f}_{0} \in \boldsymbol{B}_{\tilde{f}_{0}} \\
\tilde{\boldsymbol{d}}_{0} \in \boldsymbol{B}_{\tilde{d}_{0}}
\end{array}\right.
$$

Although accurate values of $\boldsymbol{\theta}_{i}, \tilde{\boldsymbol{f}}_{0}$, and $\tilde{\boldsymbol{d}}_{0}$ are unknown, the parameters all have definite physical meanings and they should all be bounded. $\boldsymbol{B}_{\boldsymbol{\theta}_{i}}$, $\boldsymbol{B}_{\tilde{f}_{0}}$, and $\boldsymbol{B}_{\tilde{d}_{0}}$ are the boundary restriction intervals for the unknown parameters and nonlinear uncertainties.

\section{Controller design and estimation}

The NI-PXI system is used as the physical controller and the data interactive environment from the Simulink model to embedded software (Cheng and Chen, 2013). The controller structure of the actual 3-RPS platform is shown in Fig. 3.

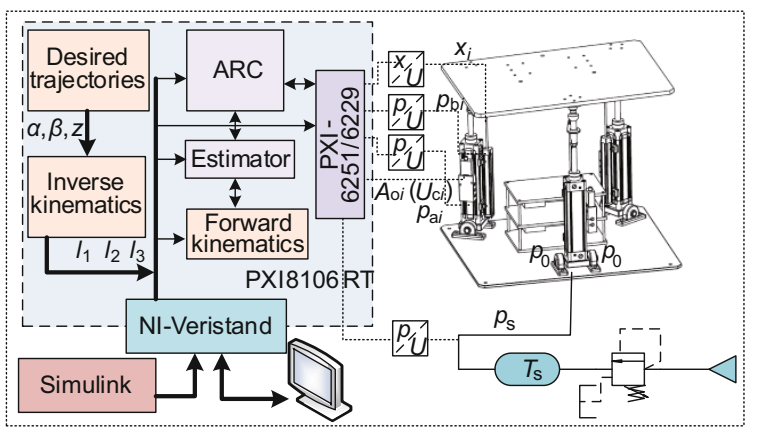

Fig. 3 Control system diagram

\subsection{Online parameter estimation}

According to the dynamic analysis of the 3-RPS platform, the unknown parameters are important for control performance. However, not all of them can be measured in advance, and the friction parameters may change as the temperature or load distribution varies. Moreover, the load feature will not be the same as in the last operation in an actual working situation. As mentioned above, given that the unknown parameters are all bounded, the least-squares method (LSM) can be used to estimate them.

Based on the parameters' online estimation, considering adaptive robust control, and assuming $\tilde{\boldsymbol{f}}_{0}=\tilde{\boldsymbol{d}}_{0}=\mathbf{0}$, multiply a stable linear time invariant (LTI) transfer function $G_{\mathrm{f}}$ with both sides of Eq. (11). Define $\boldsymbol{\varphi}_{i \mathrm{f}}^{\mathrm{T}}=G_{\mathrm{f}} \boldsymbol{\varphi}_{i}^{\mathrm{T}}$ to obtain the following equations:

$$
\left\{\begin{array}{l}
\boldsymbol{y}_{a \mathrm{f}}=G_{\mathrm{f}}\left(\boldsymbol{M}_{\mathrm{e}} \ddot{\boldsymbol{x}}-\overline{A_{\mathrm{a}}} \boldsymbol{p}_{\mathrm{L}}\right)=\boldsymbol{\varphi}_{a \mathrm{a}}^{\mathrm{T}} \boldsymbol{\theta}_{a}, \\
\boldsymbol{y}_{b \mathrm{f}}=G_{\mathrm{f}}\left(\dot{\boldsymbol{p}}_{\mathrm{L}}-\boldsymbol{q}_{\mathrm{L}}-\boldsymbol{F}_{\mathrm{p}}\right)=\boldsymbol{\varphi}_{b \mathrm{f}}^{\mathrm{T}} \boldsymbol{\theta}_{b},
\end{array}\right.
$$

which can be written as $\boldsymbol{y}_{i \mathrm{f}}=\boldsymbol{\varphi}_{i \mathrm{f}}^{\mathrm{T}} \boldsymbol{\theta}_{i}, i=a, b$ and is in the standard linear regression form. $G_{\mathrm{f}}$ is used as a filter to obtain the status values $\boldsymbol{y}_{i f}$ for the parameter estimation. According to the dynamic model of a pneumatic servo system and related studies, the transfer function's order is three. So, we set $G_{\mathrm{f}}$ as a combination of a second-order mass-spring-damping system describing the motion of the piston rod and a first-order system describing the relationship between air flow and pressure. By using this method, the calculated $\boldsymbol{y}_{i \mathrm{f}}$ is definitely stable. $G_{\mathrm{f}}$ is defined as a third-order stable transfer function with the following form:

$$
G_{\mathrm{f}}=\frac{\omega_{\mathrm{f}}^{2}}{\left(\tau_{\mathrm{f}} s+1\right)\left(s^{2}+2 \xi \omega_{\mathrm{f}} s+\omega_{\mathrm{f}}^{2}\right)} .
$$

We use $\varepsilon_{i}$ to represent the predictive error vector:

$$
\boldsymbol{\varepsilon}_{i}=\hat{\boldsymbol{y}}_{i \mathrm{f}}-\boldsymbol{y}_{i \mathrm{f}}=\boldsymbol{\varphi}_{i \mathrm{f}}^{\mathrm{T}} \hat{\boldsymbol{\theta}}_{i}-\boldsymbol{\varphi}_{i \mathrm{f}}^{\mathrm{T}} \boldsymbol{\theta}_{i}=\boldsymbol{\varphi}_{i \mathrm{f}}^{\mathrm{T}} \tilde{\boldsymbol{\theta}}_{i} .
$$

The equation above is the standard parameter estimation model. LSM can be used to obtain the estimated vector $\hat{\boldsymbol{\theta}}_{i}$.

Moreover, noncontinuous projection mapping is used to ensure that the estimation results are bounded.

$$
\dot{\hat{\boldsymbol{\theta}}}=\operatorname{sat}_{\dot{\boldsymbol{\theta}}_{\mathrm{M}}}(\underset{\hat{\boldsymbol{\theta}}}{\operatorname{Pr}}(\boldsymbol{\Psi} \boldsymbol{\tau})), \quad \hat{\boldsymbol{\theta}}(0) \in B_{\boldsymbol{\theta}},
$$

where $\boldsymbol{\Psi}$ is a positive-definite adaptive matrix, and $\boldsymbol{\tau}$ is an adaptation function. $B_{\boldsymbol{\theta}}$ is the known boundary set of the unknown parameters. $\operatorname{sat}_{\dot{\boldsymbol{\theta}}_{\mathrm{M}}}(\cdot)$ is the saturation function, which is used to limit the updating speed of the parameters. $\dot{\hat{\boldsymbol{\theta}}}_{\mathrm{M}}$ is the maximum parameter updating speed determined in advance. $\operatorname{Pr}_{\hat{\boldsymbol{\theta}}}$ is the standard projection mapping (Goodwin and Mayne, 1987; Yao and Tomizuka, 1997):

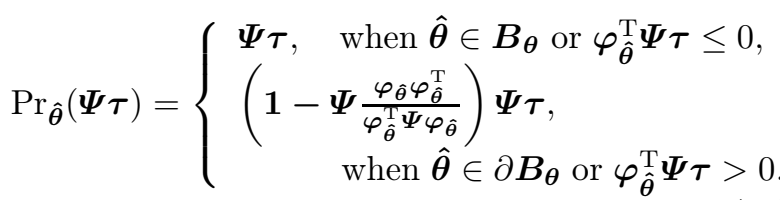

The saturation function is defined as

$$
\operatorname{sat}_{\dot{\boldsymbol{\theta}}_{\mathrm{M}}}(\cdot)=s_{a} \cdot, s_{a}= \begin{cases}1, & \|\cdot\| \leq \dot{\hat{\boldsymbol{\theta}}}_{\mathrm{M}}, \\ \dot{\hat{\boldsymbol{\theta}}}_{\mathrm{M}} /\|\cdot\|, & \|\cdot\|>\dot{\hat{\boldsymbol{\theta}}}_{\mathrm{M}} .\end{cases}
$$


Theorem 1 According to the definitions above, for any case that satisfies Eq. (16), where $\boldsymbol{\Psi}>0$ is any continuously differentiable positive symmetric adaptation rate matrix and $\boldsymbol{\tau}$ is any adaptation function, the estimated parameters vector has the properties as given below:

$$
\begin{cases}\hat{\boldsymbol{\theta}}(t) \in \boldsymbol{B}_{\boldsymbol{\theta}}=\left\{\hat{\boldsymbol{\theta}}: \boldsymbol{\theta}_{\min } \leq \hat{\boldsymbol{\theta}} \leq \boldsymbol{\theta}_{\max }\right\}, & \forall t, \\ (\hat{\boldsymbol{\theta}}-\boldsymbol{\theta})^{\mathrm{T}}\left(\boldsymbol{\Psi}^{-1} \operatorname{Pr}_{\hat{\boldsymbol{\theta}}}(\boldsymbol{\Psi} \boldsymbol{\tau})-\boldsymbol{\tau}\right) \leq 0, & \forall \boldsymbol{\tau}, \\ |\dot{\hat{\boldsymbol{\theta}}}(t)| \leq \dot{\boldsymbol{\theta}}_{\mathrm{M}}, & \forall t,\end{cases}
$$

which means that by using this approach, the estimated parameters would always be inside the closed set of $B_{\boldsymbol{\theta}}$ and the updated rate would be restricted.

The adaptive rate matrix $\boldsymbol{\Psi}_{i}$ is

$$
\dot{\boldsymbol{\Psi}}_{i}=\left\{\begin{array}{c}
\alpha_{i} \boldsymbol{\Psi}_{i}-\frac{\boldsymbol{\Psi}_{i} \boldsymbol{\varphi}_{i \mathrm{f}} \boldsymbol{\varphi}_{i \mathrm{~T}}^{\mathrm{T}} \boldsymbol{\Psi}_{i}}{1+\nu_{i} \boldsymbol{\varphi}_{i \mathrm{f}}^{\mathrm{T}} \boldsymbol{\Psi}_{i} \boldsymbol{\varphi}_{i \mathrm{f}}}, \\
\text { when } \lambda_{\max }\left(\boldsymbol{\Psi}_{i}(t)\right) \leq \rho_{\mathrm{M} i} \\
\text { and }\left\|\operatorname{Pr}_{\hat{\boldsymbol{\theta}}}\left(\Psi_{i} \boldsymbol{\tau}_{i}\right)\right\| \leq \hat{\boldsymbol{\theta}}_{\mathrm{M} i}, \\
\mathbf{0}, \quad \text { otherwise, }
\end{array}\right.
$$

where $1 \geq \alpha_{i} \geq 0$ is the fading factor that reduces the influence caused by the old data. $\nu \geq 0$ is the normalized factor, $\lambda_{\max }\left(\boldsymbol{\Psi}_{i}(t)\right)$ is the largest eigenvalue of $\boldsymbol{\Psi}_{i}(t)$, and $\rho_{\mathrm{M} i}$ is the upper bound of $\left\|\boldsymbol{\Psi}_{i}(t)\right\|$. $\boldsymbol{\tau}_{i}$ is the adaptive function, as shown in Eq. (21):

$$
\boldsymbol{\tau}_{i}=\frac{1}{1+\nu_{i} \boldsymbol{\varphi}_{i \mathrm{f}}^{\mathrm{T}} \boldsymbol{\Psi}_{i} \boldsymbol{\varphi}_{i \mathrm{f}}} \boldsymbol{\varphi}_{i \mathrm{f}} \boldsymbol{\varepsilon}_{i}
$$

\subsection{Load compensation}

Determination of the parameters' initial values can help the system status converge into a specific stable condition faster, compared with nonchangeable initial factors. To estimate these numbers, identification of the dynamic parameters of the 3-RPS system can be helpful (Atkeson et al., 1985; Grotjahn et al., 2004; Farhat et al., 2008).

According to the Gibbs-Appell equations, the open-chain mechanical system dynamics can be written in a linear form as

$$
\boldsymbol{W}(\boldsymbol{q}, \dot{\boldsymbol{q}}, \ddot{\boldsymbol{q}}) \cdot \boldsymbol{\Phi}=\tau_{\mathrm{L}}
$$

where $\boldsymbol{W}$ is a matrix that has the size $n_{\text {dof }} \times(10 n+$ $\left.n_{\mathrm{fr}}\right) . n_{\mathrm{fr}}$ is the number of frictional model parameters, and $n$ is the number of moving parts. $\tau_{\mathrm{L}}$ is the generalized forces vector applied to the actuators. $\boldsymbol{\Phi}$ is the vector of the dynamic parameters. If we focus only on the upper moving board, then $n=1$.

$$
\boldsymbol{\Phi}=\left[\begin{array}{lll}
\boldsymbol{M}_{\boldsymbol{\Phi}} & \boldsymbol{I}_{\boldsymbol{\Phi}} & \boldsymbol{\Phi}_{\mathrm{fri}}
\end{array}\right]^{\mathrm{T}},
$$

where $\boldsymbol{M}_{\boldsymbol{\Phi}}=\left[m_{i}, m c_{x i}, m c_{y i}, m c_{z i}\right], m_{i}$ is the mass of each body, and $m c_{x i}, m c_{y i}$, and $m c_{z i}$ are elements of the first mass moment with respect to the body's mass center. $\boldsymbol{I}_{\boldsymbol{\Phi}}$ are the values of each body's inertia tensor elements, calculated about its local reference. $\boldsymbol{\Phi}_{\text {fri }}$ are the frictional model parameters.

In matrix $\boldsymbol{W}$, there are many zero columns that have no effect on the dynamic system as a whole, if we consider all moving parts in the 3-RPS structure. There are also some columns that have the same direction as the others, which can also be reduced. Thus, the observation matrix $\boldsymbol{W}$ is rank-deficient and there are an infinite number of parameter solutions. As such, it is important to reduce the size of the dynamic model matrix to a smaller one that is sufficient to solve for the parameters. Moreover, considering the dynamic model reduction and the frictional characteristics, the dynamic model of the system has the following form:

$$
\boldsymbol{W}_{\mathrm{r}}^{\mathrm{I}} \cdot \boldsymbol{\Phi}_{\mathrm{b}}^{\mathrm{I}}+\left(\boldsymbol{F}_{\boldsymbol{f}_{\mathrm{i}}}-\boldsymbol{Z}^{\mathrm{T}} \boldsymbol{F}_{\boldsymbol{f}_{\mathrm{e}}}\right)=\boldsymbol{\tau}_{\mathrm{L}}
$$

where $\boldsymbol{W}_{\mathrm{r}}^{\mathrm{I}}$ is the reduced observation matrix of the inertia part, and $\boldsymbol{\Phi}_{\mathrm{b}}^{\mathrm{I}}$ is the base parameter, equal to $\operatorname{rank}\left(\boldsymbol{W}_{\mathrm{r}}^{\mathrm{I}}\right) . \boldsymbol{F}_{\boldsymbol{f}_{\mathrm{i}}}$ and $\boldsymbol{F}_{\boldsymbol{f}_{\mathrm{e}}}$ are independent and dependent friction forces, respectively.

Based on the theory above, before estimating the initial values of some key elements and applying them to the real situation in this study, some processes still need to be addressed. The main object is the moving platform; the influence of the cylinders and pistons is ignored to decrease the size of the observation matrix and the parameter vector. Because the main friction forces exist in the sliding pairs between the cylinder walls and pistons, all other friction forces on spherical and revolute joints can be ignored; i.e., $\boldsymbol{F}_{\boldsymbol{f}_{\mathrm{e}}}$ is regarded as zero. The external forces and torque applied to the upper board, i.e., $\boldsymbol{F}_{\mathrm{Be}}$ and $\boldsymbol{\tau}_{\mathrm{B}}$, have the form shown in Eqs. (25) and (26):

$$
\begin{aligned}
& \boldsymbol{F}_{\mathrm{Be}}=\boldsymbol{F}_{\mathrm{Bd}}-\boldsymbol{F}_{\mathrm{fs}} \\
& =m \ddot{\boldsymbol{P}}_{\boldsymbol{O}_{\mathrm{B}}}-m \boldsymbol{g}+\dot{\boldsymbol{\omega}}_{\boldsymbol{O}_{\mathrm{B}}} \times m \boldsymbol{c}+\boldsymbol{\omega}_{\boldsymbol{O}_{\mathrm{B}}} \times\left(\boldsymbol{\omega}_{\boldsymbol{O}_{\mathrm{B}}} \times m \boldsymbol{c}\right), \\
& \boldsymbol{\tau}_{\mathrm{B}}=\boldsymbol{I}_{\boldsymbol{O}_{\mathrm{B}}} \dot{\boldsymbol{\omega}}_{\boldsymbol{O}_{\mathrm{B}}}+\dot{\boldsymbol{\omega}}_{\boldsymbol{O}_{\mathrm{B}}} \times\left(\boldsymbol{I}_{\boldsymbol{O}_{\mathrm{B}}} \boldsymbol{\omega}_{\boldsymbol{O}_{\mathrm{B}}}\right) \\
& \quad+m \boldsymbol{c} \times\left(\dot{\boldsymbol{\omega}}_{\boldsymbol{O}_{\mathrm{B}}} \times \boldsymbol{c}\right)+m \boldsymbol{c} \times\left(\boldsymbol{\omega}_{\boldsymbol{O}_{\mathrm{B}}} \times\left(\boldsymbol{\omega}_{\boldsymbol{O}_{\mathrm{B}}} \times \boldsymbol{c}\right)\right) \\
& \quad+m \boldsymbol{c} \times \ddot{\boldsymbol{P}}_{\boldsymbol{O}_{\mathrm{B}}}-m \boldsymbol{c} \times \boldsymbol{g},
\end{aligned}
$$

where $\boldsymbol{F}_{\mathrm{Bd}}$ can be detected by pressure sensors attached to all chambers. $\boldsymbol{F}_{\mathrm{fs}}$ is the sliding friction 
force. $\boldsymbol{P}_{\boldsymbol{O}_{\mathrm{B}}}$ is the origin of the moving coordinate attached to the moving board in global coordinates. $\boldsymbol{g}$ is the vector of gravity, which equals $[0,0,-9.8] \mathrm{m} / \mathrm{s}^{2}$. $c$ is the unknown location of the mass center relative to $\boldsymbol{P}_{\boldsymbol{O}_{\mathrm{B}}} \cdot \boldsymbol{\omega}_{\boldsymbol{O}_{\mathrm{B}}}$ and $\dot{\boldsymbol{\omega}}_{\boldsymbol{O}_{\mathrm{B}}}$ are the angular velocity vector and angular acceleration vector, respectively.

The friction model is described by the following equations:

$$
\left\{\begin{array}{l}
\boldsymbol{f}_{\mathrm{s} i}=\left(b_{i} \dot{x}_{i}+A_{\mathrm{f} i} S_{\mathrm{f} i}\left(\dot{x}_{i}\right)\right) \boldsymbol{n}_{i} \\
\boldsymbol{F}_{\mathrm{fs}}=\sum \boldsymbol{f}_{\mathrm{s} i}
\end{array}\right.
$$

where $\boldsymbol{n}_{i}$ is the normalized direction vector of each piston rod, which can be calculated based on the position data of all cylinders.

Combining Eqs. (25)-(27), we obtain

$$
\boldsymbol{U}_{\mathrm{c}}^{\mathrm{T}}=\left[\boldsymbol{F}_{\mathrm{Bd}}^{\mathrm{T}}, \boldsymbol{\tau}_{\mathrm{B}}^{\mathrm{T}}\right]^{\mathrm{T}}=\boldsymbol{W}_{\mathrm{c}}^{\mathrm{T}} \boldsymbol{\Phi}_{\mathrm{c}}
$$

where $\boldsymbol{W}_{\mathrm{c}}^{\mathrm{T}}$ is the observation matrix and $\boldsymbol{\Phi}_{\mathrm{c}}$ is the parameter vector.

$$
\boldsymbol{W}_{\mathrm{c}}^{\mathrm{T}}=\left[\begin{array}{cccc}
\boldsymbol{P}_{\boldsymbol{W}} & \boldsymbol{C}_{\boldsymbol{W} 1} & \mathbf{0} & \boldsymbol{N} \cdot \boldsymbol{B} \\
\mathbf{0} & \boldsymbol{C}_{\boldsymbol{W} 2} & \boldsymbol{C}_{\boldsymbol{W} P} & \mathbf{0}
\end{array}\right] .
$$

The calculation equations for these elements in $\boldsymbol{W}_{\mathrm{c}}^{\mathrm{T}}$ are shown in Eqs. (30)-(33). $\quad \boldsymbol{P}_{\boldsymbol{W}}=\ddot{\boldsymbol{P}}_{\boldsymbol{O}_{\mathrm{B}}}-$ g. $C_{W 1}=\boldsymbol{C}\left(\dot{\omega}_{O_{\mathrm{B}}}\right)+\boldsymbol{C}\left(\boldsymbol{\omega}_{O_{\mathrm{B}}}\right) \boldsymbol{C}\left(\boldsymbol{\omega}_{O_{\mathrm{B}}}\right), \boldsymbol{C}_{\boldsymbol{W} 2}=$ $\boldsymbol{C}\left(\ddot{\boldsymbol{P}}_{\boldsymbol{O}_{\mathrm{B}}}-\boldsymbol{g}\right), \boldsymbol{C}_{\boldsymbol{W} P}=\boldsymbol{D}\left(\dot{\boldsymbol{\omega}}_{\boldsymbol{O}_{\mathrm{B}}}\right)+\boldsymbol{C}\left(\boldsymbol{\omega}_{\boldsymbol{O}_{\mathrm{B}}}\right) \boldsymbol{D}\left(\boldsymbol{\omega}_{\boldsymbol{O}_{\mathrm{B}}}\right)$.

$$
\begin{aligned}
& \left\{\begin{array}{l}
\boldsymbol{N}=\left[\boldsymbol{n}_{1}, \boldsymbol{n}_{2}, \boldsymbol{n}_{3}\right], \\
\boldsymbol{B}=\left[\operatorname{diag}\left(\dot{x}_{1}, \dot{x}_{2}, \dot{x}_{3}\right), \operatorname{diag}\left(S_{\mathrm{c} 1}, S_{\mathrm{c} 2}, S_{c 3}\right)\right]_{3 \times 6},
\end{array}\right. \\
& \boldsymbol{C}(a)=\left[\begin{array}{ccc}
0 & -a_{z} & a_{y} \\
a_{z} & 0 & -a_{x} \\
-a_{y} & a_{x} & 0
\end{array}\right] \\
& \boldsymbol{D}(a)=\left[\begin{array}{cccccc}
a_{x} & a_{y} & a_{z} & 0 & 0 & 0 \\
0 & a_{x} & 0 & a_{y} & a_{z} & 0 \\
0 & 0 & a_{x} & 0 & a_{y} & a_{z}
\end{array}\right] \\
& \boldsymbol{I}_{\boldsymbol{O}_{\mathrm{B}}}=\boldsymbol{I}_{\boldsymbol{O}_{\mathrm{B}}}^{\mathrm{T}}=\left[\begin{array}{ccc}
I_{11} & I_{12} & I_{13} \\
I_{21} & I_{22} & I_{23} \\
I_{31} & I_{32} & I_{33}
\end{array}\right] \text {. }
\end{aligned}
$$

\subsection{Controller structure}

Based on back-stepping concepts, there are two steps in designing the controller, by which the calculated control values are finally exported for the proportional valves. The first step is considered at the level of pressure. $\boldsymbol{e}_{\mathrm{S}}$ is defined as a vector that is similar to the sliding model surface vector. $\boldsymbol{K}_{\boldsymbol{x}}$ is the constant matrix chosen as a proportional feedback of the tracking error vector $\boldsymbol{e}=\boldsymbol{x}-\boldsymbol{x}_{\mathrm{d}}$.

$$
\left\{\begin{array}{l}
e_{\mathrm{s}}=\dot{e}+\boldsymbol{K}_{x} e \\
\dot{e}_{\mathrm{s}}=\ddot{e}+\boldsymbol{K}_{x}\left(\dot{\boldsymbol{x}}-\dot{\boldsymbol{x}}_{\mathrm{d}}\right)
\end{array}\right.
$$

$\boldsymbol{p}_{\mathrm{L}}$ is used as the first-level virtual input, and $\boldsymbol{e}_{\mathrm{p}}=\boldsymbol{p}_{\mathrm{L}}-\boldsymbol{p}_{\mathrm{Ld}}$ is used to describe the error between the desired pressure vector $\boldsymbol{p}_{\mathrm{Ld}}$ and the measured pressure vector $\boldsymbol{p}_{\mathrm{L}}$.

$$
\boldsymbol{p}_{\mathrm{Ld}}=\boldsymbol{p}_{\mathrm{Lda} 1}+\boldsymbol{p}_{\mathrm{Lda} 2}+\boldsymbol{p}_{\mathrm{Lds} 1}+\boldsymbol{p}_{\mathrm{Lds} 2}
$$

where $\boldsymbol{p}_{\text {Lda1 }}$ is the model compensation part and $\boldsymbol{p}_{\mathrm{Lds} 1}$ is chosen as the proportional feedback to stabilize the ideal modeled dynamic system:

$$
\left\{\begin{array}{l}
\boldsymbol{p}_{\mathrm{Lda} 1}=\left(-\boldsymbol{\varphi}_{a}^{\mathrm{T}} \hat{\boldsymbol{\theta}}_{a}+\boldsymbol{M}_{\mathrm{e}} \ddot{\boldsymbol{x}}_{\mathrm{d}}-\boldsymbol{M}_{\mathrm{e}} \boldsymbol{K}_{\boldsymbol{x}} \boldsymbol{e}\right) / \bar{A}_{a} \\
\boldsymbol{p}_{\mathrm{Lds} 1}=-\boldsymbol{K}_{\mathrm{p}} \boldsymbol{e}_{\mathrm{s}} / \bar{A}_{a}
\end{array}\right.
$$

where $\boldsymbol{K}_{\mathrm{p}}$ is a positive-definite diagonal matrix. $\boldsymbol{p}_{\mathrm{Lda} 2}$ is the fast compensation part. $\boldsymbol{p}_{\mathrm{Lds} 2}$ is the robust feedback part that inhibits the influence caused by the parameter estimation error and uncertain nonlinearity. This part can be defined as

$$
\left\{\begin{array}{l}
\boldsymbol{d}_{\mathrm{c} 1}+\boldsymbol{R}_{1}(t)=-\boldsymbol{\varphi}_{a}^{\mathrm{T}} \tilde{\boldsymbol{\theta}}_{a}+\tilde{\boldsymbol{f}}_{0} \\
\boldsymbol{p}_{\mathrm{Lda} 2}=-\hat{\boldsymbol{d}}_{\mathrm{c} 1} / \bar{A}_{a}
\end{array}\right.
$$

where $\boldsymbol{d}_{\mathrm{c} 1}$ is the low-frequency part of the estimation error and $\boldsymbol{R}_{1}(t)$ is the high-frequency part.

Theorem $2 \boldsymbol{d}_{\mathrm{c} 1}$ can be estimated readily by defining $\hat{\boldsymbol{d}_{\mathrm{c} 1}}=\operatorname{Pr}_{\hat{\boldsymbol{d}_{\mathrm{c} 1}}}\left(\boldsymbol{\gamma}_{1} \boldsymbol{e}_{\mathrm{s}}\right)$ and is used to calculate $\boldsymbol{p}_{\mathrm{Lda} 2}$.

$$
\begin{aligned}
& \operatorname{Pr}_{\hat{\boldsymbol{d}}_{\mathrm{c} 1}}\left(\gamma_{1} \boldsymbol{e}_{\mathrm{s}}\right) \\
& =\left\{\begin{array}{l}
0, \text { when }\left\|\hat{\boldsymbol{d}}_{\mathrm{c} 1}\right\|=d_{\mathrm{c} 1 \mathrm{M}} \text { or } \hat{\boldsymbol{d}}_{\mathrm{c} 1} \boldsymbol{e}_{\mathrm{s}}>0, \\
\gamma_{1} \boldsymbol{e}_{\mathrm{s}}, \text { else. }
\end{array}\right.
\end{aligned}
$$

$\gamma_{1}$ is a positive diagonal matrix and $\gamma_{1 i}$ is the adaptive rate value of each $d_{\mathrm{c} 1 i}$, where $i=1,2,3$. According to Theorem 1 and Eq. (37), $\boldsymbol{p}_{\mathrm{Lds} 2}$ is defined by

$$
\begin{gathered}
\boldsymbol{p}_{\mathrm{Lds} 2}=-\frac{1}{\bar{A}_{a}} \frac{H_{1}^{2}(t)}{4 \eta_{1}} \boldsymbol{e}_{\mathrm{s}}, \\
H_{1}(t) \geq d_{\mathrm{c} 1 \mathrm{M}}+\left\|\boldsymbol{\theta}_{\mathrm{M} a}\right\|\left\|\boldsymbol{\varphi}_{a}\right\|+f_{\max },
\end{gathered}
$$

where $d_{\mathrm{c} 1 \mathrm{M}}$ is the upper bound of $\left\|\boldsymbol{d}_{\mathrm{c} 1}\right\|$ and $\boldsymbol{\theta}_{\mathrm{M} a}=$ $\boldsymbol{\theta}_{\max a}-\boldsymbol{\theta}_{\min a}$. 
The purpose of the second-level controller design is to make $\boldsymbol{e}_{\mathrm{p}}=\boldsymbol{p}_{\mathrm{L}}-\boldsymbol{p}_{\mathrm{Ld}}$ converge to zero. Then, the derivation of $\boldsymbol{e}_{\mathrm{p}}$ is given by

$$
\dot{e}_{\mathrm{p}}=\boldsymbol{q}_{\mathrm{L}}+\boldsymbol{F}_{\mathrm{p}}+\boldsymbol{\varphi}_{b}^{\mathrm{T}} \boldsymbol{\theta}_{b}+\tilde{\boldsymbol{d}}_{0}-\dot{\boldsymbol{p}}_{\mathrm{Ld}} .
$$

Eq. (40) indicates that this step is considered in the level of flow rate. Because the flow rate is determined by the control voltage directly, the result of this step can be used in the calculation of the control value vector $\boldsymbol{u}_{\mathrm{c}}$.

$$
\boldsymbol{q}_{\mathrm{L}}=\boldsymbol{q}_{\mathrm{Lda} 1}+\boldsymbol{q}_{\mathrm{Lda} 2}+\boldsymbol{q}_{\mathrm{Lds} 1}+\boldsymbol{q}_{\mathrm{Lds} 2} .
$$

Similar to the first step of the controller design of $\boldsymbol{p}_{\mathrm{L}}, \boldsymbol{q}_{\mathrm{Lda} 1}$ is the model compensation part, and $\boldsymbol{q}_{\mathrm{Lds} 1}$ is chosen as the proportional feedback to stabilize the ideal modeled dynamic system:

$$
\left\{\begin{array}{l}
\boldsymbol{q}_{\mathrm{Lda} 1}=-\bar{A}_{a} \boldsymbol{e}_{\mathrm{s}}-\boldsymbol{F}_{\mathrm{p}}-\boldsymbol{\varphi}_{b}^{\mathrm{T}} \hat{\boldsymbol{\theta}}_{b}+\dot{\boldsymbol{p}}_{\mathrm{Ld}} \\
\boldsymbol{q}_{\mathrm{Lds} 1}=-\boldsymbol{K}_{\mathrm{q}} \boldsymbol{e}_{\mathrm{p}}
\end{array}\right.
$$

where $\boldsymbol{K}_{\mathrm{q}}$ is a positive-definite diagonal matrix. $\boldsymbol{q}_{\mathrm{Lda} 2}$ is the fast compensation part. $\boldsymbol{q}_{\mathrm{Lds} 2}$ is the robust feedback part that inhibits the influence caused by the parameter estimation error and uncertain nonlinearity. This part can be defined as

$$
\left\{\begin{array}{l}
\boldsymbol{d}_{\mathrm{c} 2}+\boldsymbol{R}_{2}(t)=-\boldsymbol{\varphi}_{b}^{\mathrm{T}} \tilde{\boldsymbol{\theta}}_{b}+\tilde{\boldsymbol{d}}_{0}, \\
\boldsymbol{q}_{\mathrm{Lda} 2}=-\hat{\boldsymbol{d}}_{\mathrm{c} 2}
\end{array}\right.
$$

where $\boldsymbol{d}_{\mathrm{c} 2}$ is the low-frequency part of the estimation error and $\boldsymbol{R}_{2}(t)$ is the high-frequency part.

Theorem $3 \quad d_{\mathrm{c} 2}$ can be estimated readily by defining $\hat{\boldsymbol{d}_{\mathrm{c} 2}}=\operatorname{Pr}_{\hat{\mathrm{c}^{2}}}\left(\gamma_{2} \boldsymbol{e}_{\mathrm{p}}\right)$ and is used to calculate $\boldsymbol{q}_{\mathrm{Lda} 2}$.

$$
\begin{aligned}
& \operatorname{Pr}_{\hat{\boldsymbol{d}}_{\mathrm{c} 2}}\left(\gamma_{2} \boldsymbol{e}_{\mathrm{p}}\right) \\
& =\left\{\begin{array}{l}
0, \text { when }\left\|\hat{\boldsymbol{d}}_{\mathrm{c} 2}\right\|=d_{\mathrm{c} 2 \mathrm{M}} \text { or } \hat{\boldsymbol{d}}_{\mathrm{c} 2} \boldsymbol{e}_{\mathrm{p}}>0, \\
\gamma_{2} \boldsymbol{e}_{\mathrm{p}}, \text { else. }
\end{array}\right.
\end{aligned}
$$

$\gamma_{2}$ is a positive diagonal matrix and $\gamma_{2 i}$ is the adaptive rate value of each $d_{\mathrm{c} 2 i}$, where $i=1,2,3$. According to Theorem 1 and Eq. (43), $\boldsymbol{q}_{\mathrm{Lds} 2}$ is defined by

$$
\begin{gathered}
\boldsymbol{q}_{\mathrm{Lds} 2}=-\frac{H_{2}^{2}(t)}{4 \eta_{2}} \boldsymbol{e}_{\mathrm{p}} \\
H_{2}(t) \geq d_{\mathrm{c} 2 \mathrm{M}}+\left\|\boldsymbol{\theta}_{\mathrm{M} b}\right\|\left\|\boldsymbol{\varphi}_{b}\right\|+\boldsymbol{d}_{\max }
\end{gathered}
$$

where $d_{\mathrm{c} 2 \mathrm{M}}$ is the upper bound of $\left\|\boldsymbol{d}_{\mathrm{c} 2}\right\|$ and $\boldsymbol{\theta}_{\mathrm{M} b}=$ $\boldsymbol{\theta}_{\max b}-\boldsymbol{\theta}_{\min b}$.
Finally, when $\boldsymbol{q}_{\mathrm{Ld}}$ is calculated, the inverse processing of flow rate calculation is applied according to Eq. (47):

$$
\begin{aligned}
& A_{i}(u)= \\
& \left\{\begin{array}{cc}
H_{\mathrm{p}} \boldsymbol{q}_{\mathrm{Ld} i} /\left(\gamma_{\mathrm{a}} R T_{\mathrm{s}} K_{\mathrm{q}}\left(\boldsymbol{p}_{\mathrm{s}}, \boldsymbol{p}_{a}, T_{\mathrm{s}}\right) / V_{a}\right. & \\
\left.+\gamma_{\mathrm{a}} R T_{b} K_{\mathrm{q}}\left(\boldsymbol{p}_{b}, \boldsymbol{p}_{0}, T_{b}\right) k_{a} / V_{\mathrm{b}}\right), & \boldsymbol{q}_{\mathrm{Ld} i}>0, \\
H_{\mathrm{p}} \boldsymbol{q}_{\mathrm{Ld} i} /\left(-\gamma_{\mathrm{a}} R T_{a} K_{\mathrm{q}}\left(\boldsymbol{p}_{a}, \boldsymbol{p}_{0}, T_{\mathrm{s}}\right) / V_{a}\right. & \\
\left.-\gamma_{\mathrm{a}} R T_{\mathrm{s}} K_{\mathrm{q}}\left(\boldsymbol{p}_{\mathrm{s}}, \boldsymbol{p}_{b}, T_{b}\right) k_{a} / V_{b}\right), & \boldsymbol{q}_{\mathrm{Ld} i}<0 .
\end{array}\right.
\end{aligned}
$$

Determined by the valve testing experiment results shown in Fig. 4, the relationship between the control voltage and valve area can be expressed as

$$
\begin{aligned}
J\left(u_{\mathrm{c} i}\right) & = \begin{cases}K_{A} u_{\mathrm{cd} i}^{2}+K_{B} u_{\mathrm{cd} i}+K_{C}, & \left|u_{\mathrm{cd} i}\right| \geq u_{\mathrm{d} z i}, \\
\frac{K_{D} u_{\mathrm{cd} i}+K_{E}}{K_{F} u_{\mathrm{cd} i}+K_{G}}, & \left|u_{\mathrm{cd} i}\right|<u_{\mathrm{dz} i},\end{cases} \\
u_{\mathrm{cd} i} & =\left(u_{\mathrm{c} i}-u_{\mathrm{m} i}\right) / 10,
\end{aligned}
$$

where $u_{\mathrm{cd} i}, u_{\mathrm{m} i}, u_{\mathrm{c} i}$, and $u_{\mathrm{dz} i}$ are normalized control values, middle control values, real output voltage, and dead zone values, respectively, and $K_{A}-K_{G}$ are constants to describe the flow rate curve, shown in Fig. 4. Then, the real output voltage $\boldsymbol{u}_{\mathrm{c}}$ is given by

$$
u_{\mathrm{c} i}=J^{-1}\left(A_{i}(u)\right) .
$$

The stability proof of this controller design is given in the Appendix.

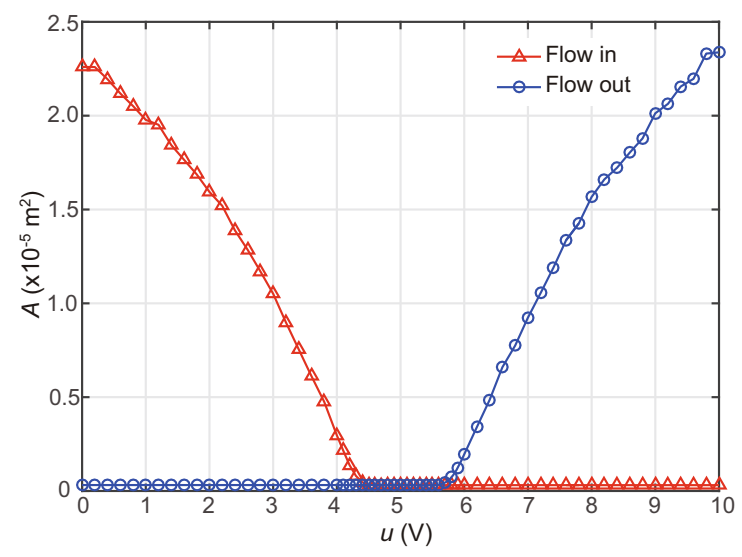

Fig. 4 FESTO MPYE 1/4' valve model

\subsection{Controller simulation}

Using the configurations shown in Table 1, a model of the 3-RPS pneumatic platform was built in Simulink. The unknown parameters 
were initialized as $\hat{\boldsymbol{\theta}}_{i}=[100,20,10]^{\mathrm{T}}, \boldsymbol{\theta}_{b i}=\mathbf{0}$, $\boldsymbol{\rho}_{\mathrm{M} i}=[1000,100,100,1000,100,100,1000,100,100]$, $\dot{\boldsymbol{\theta}}_{\mathrm{M} i}=[10,10,10,10,10,10,10,10,10]^{\mathrm{T}}, \quad \dot{\boldsymbol{\theta}}_{\mathrm{M} b i}=$ $[10,10,10]^{\mathrm{T}}$. The desired trajectory is a sine wave on each axis. The controller ran for $40 \mathrm{~s}$. The posture tracking performance is shown in Fig. 5. This simulation confirmed the effectiveness of the online parameter estimator and the performance of the ARC controller.

Table 1 Constant values used in the model

\begin{tabular}{clc}
\hline Symbol & \multicolumn{1}{c}{ Description } & Value \\
\hline$A_{\mathrm{a}}$ & Chamber $A$ area & $3.12 \times 10^{-3} \mathrm{~m}^{2}$ \\
$A_{b}$ & Chamber $B$ area & $2.803 \times 10^{-3} \mathrm{~m}^{2}$ \\
$R$ & Air constant & $287 \mathrm{~N} \cdot \mathrm{m} /(\mathrm{kg} \cdot \mathrm{K})$ \\
$T_{\mathrm{s}}$ & Source temperature & $295 \mathrm{~K}$ \\
$L$ & Cylinder length & $0.2 \mathrm{~m}$ \\
$\gamma_{\mathrm{a}}$ & Heat capacity ratio & 1.4 \\
$\tau_{\mathrm{f}}, \omega_{\mathrm{f}}, \xi_{\mathrm{f}}$ & Filter parameters & $1,50,0.1$ \\
$V_{\mathrm{o} i}$ & Dead volume & $0.2 \mathrm{~L}$ \\
$h$ & Heat-trans constant & $50 \mathrm{~W} /\left(\mathrm{m}{ }^{2} \cdot \mathrm{K}\right)$ \\
$D$ & Cylinder diameter & $0.063 \mathrm{~m}$ \\
$\alpha_{i}$ & Fading factor & 0.1 \\
$\nu_{i}$ & Normalization factor & 0.1 \\
$H_{1}(t), \eta_{1}$ & $\boldsymbol{p}_{\text {Lds2 }}$ constant & 80,4 \\
$H_{2}(t), \eta_{2}$ & $\boldsymbol{q}_{\mathrm{Lds} 2}$ constant & 200,10 \\
$\boldsymbol{K}_{\boldsymbol{x}}$ & Error vector feedback & $\operatorname{diag}(45,45,45)$ \\
$\boldsymbol{K}_{\mathrm{p}}$ & $\boldsymbol{p}_{\text {Lds1 feedback }}$ & $\operatorname{diag}(20,20,20)$ \\
$\boldsymbol{K}_{\mathrm{q}}$ & $\boldsymbol{q}_{\mathrm{Lds} 1 \text { feedback }}$ & $\operatorname{diag}(60,60,60)$ \\
$u_{\mathrm{dz}}$ & Dead-zone voltage & $0.64 \mathrm{~V}$ \\
$u_{\mathrm{c}}$ & Valve center voltage & $5.02 \mathrm{~V}$ \\
$\Psi_{i}(0)_{j j}$ & $j=1,2, \cdots, 9$ & 100 \\
\hline & &
\end{tabular}
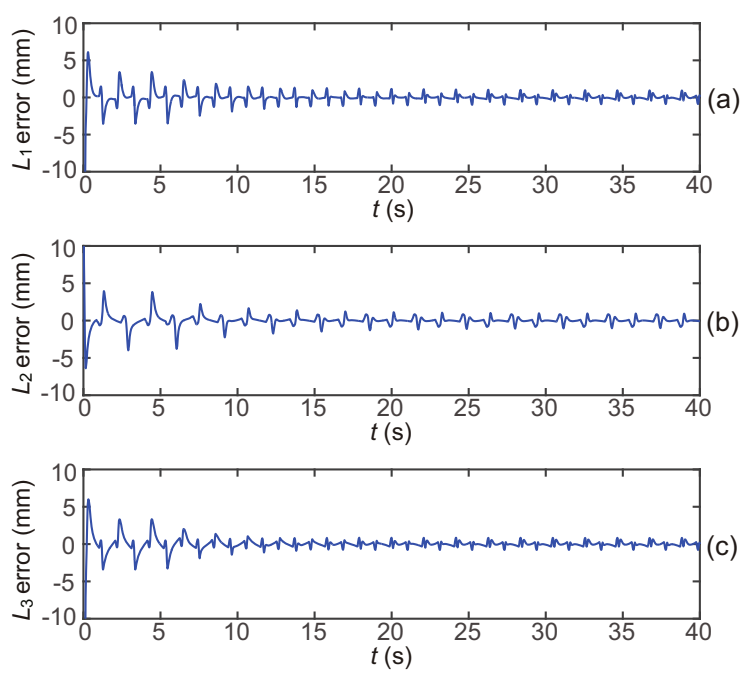

Fig. 5 Simulation results of the tracking error: (a) $L_{1}$ error; (b) $L_{2}$ error; (c) $L_{3}$ error

\section{Experiment results}

We programmed the controller designed in the previous section and applied it to the real hardware (Fig. 2). Different from the simulation, all the experiments were executed on the actual plant to verify the designed controller's performance. A brief structure of the hardware system is shown in Fig. 3. The controller was established using Matlab/Simulink software, and Realtime-Workshop was used to build the embedded file, which could be downloaded into the PXI system. NI-Veristand was used as the datainteractive environment between the host-PC (dashboard) and real-time controller (NI-PXI8106 RT).

Each independent experiment consisted of three steps: initial parameter identification, trajectory tracking control, and error analysis.

The first step solves the problems with obvious changes in the load, temperature, or a long time interval since the last experiment. In those situations, the parameters are regarded as unknown and changeable. To improve the control performance, compared to constant initial configurations, a better set of initial values of the unknown parameters should be determined first. If there is no significant difference in the external influence, such as load and temperature, between the two times of trajectory tracking control, and the second control experiment is executed immediately after the first one, then there is no need to estimate the initial values of the parameters again. The former settings can still be applied until the load features change.

During the first process, the controller must execute the configuration process of the initial values. The trajectory is a compound sine wave and the points are selected at the smoothing moving status while the system is under the control of a PID controller. After selecting a series of estimation points, the matrices $\left(\boldsymbol{U}_{\mathrm{c}}^{\mathrm{T}}\right)_{6 \mathrm{npts} \times 1}$ and $\left(\boldsymbol{W}_{\mathrm{c}}^{\mathrm{T}}\right)_{6 \mathrm{npts} \times 16}$ in Eq. (28) are built. Then, the parameter vector $\left(\boldsymbol{\Phi}_{\mathrm{c}}\right)_{16 \times 1}$ can be calculated:

$$
\boldsymbol{\Phi}_{\mathrm{c}}=\frac{\boldsymbol{W}_{\mathrm{c}} \boldsymbol{U}_{\mathrm{c}}^{\mathrm{T}}}{\boldsymbol{W}_{\mathrm{c}}^{\mathrm{T}} \boldsymbol{W}_{\mathrm{c}}} .
$$

According to Eq. (8), set $\boldsymbol{\theta}_{\mathrm{o} i}$ as the initial parameter vector, $\boldsymbol{\theta}_{\mathrm{o} i}=\left[b_{\mathrm{o} i}, A_{\mathrm{of} i},-F_{\mathrm{oL} i}+f_{\mathrm{on} i}\right], i=$ $1,2,3$, and $-F_{\mathrm{oL} i}+f_{\mathrm{on} i}$ is expressed as $\theta_{\mathrm{o} i 3}, i=$ $1,2,3 . \quad-F_{\mathrm{L} i}+f_{\mathrm{n} i}$ can be calculated using an inverse force Jacobian reflection, as shown in Eq. (51). 
Then, the parameter vectors are initialized. $\boldsymbol{J}_{\mathrm{f}}$ is the Jacobian force matrix. Table 2 shows the identification results for the dynamic initial parameters of the system.

$$
\left(-\boldsymbol{F}_{\mathrm{L}}+\boldsymbol{f}_{\mathrm{n}}\right)_{3 \times 1}=\left(\boldsymbol{J}_{\mathrm{f}}^{-1}[m \boldsymbol{g}, m \boldsymbol{c} \times \boldsymbol{g}]^{\mathrm{T}}\right)_{3 \times 1} .
$$

Table 2 Initial value estimation results

\begin{tabular}{cc||cc}
\hline Parameter & Value & Parameter & Value \\
\hline$m$ & $24.50 \mathrm{~kg}$ & $b_{\mathrm{o} 1}$ & 412 \\
$m c_{x}$ & $0.3 \mathrm{~kg} \cdot \mathrm{m}$ & $b_{\mathrm{O} 2}$ & 327 \\
$m c_{y}$ & $3.8 \mathrm{~kg} \cdot \mathrm{m}$ & $b_{\mathrm{O} 3}$ & 345 \\
$m c_{z}$ & $0.5 \mathrm{~kg} \cdot \mathrm{m}$ & $A_{\mathrm{of} 1}$ & 20.8 \\
$I_{11}$ & $1.44 \mathrm{~kg} \cdot \mathrm{m}^{2}$ & $A_{\mathrm{of} 2}$ & 19.5 \\
$I_{12}$ & $0.12 \mathrm{~kg} \cdot \mathrm{m}^{2}$ & $A_{\mathrm{of} 3}$ & 22.3 \\
$I_{13}$ & $0.09 \mathrm{~kg} \cdot \mathrm{m}^{2}$ & $\theta_{\mathrm{O} 13}$ & $-112 \mathrm{~N}$ \\
$I_{22}$ & $0.95 \mathrm{~kg} \cdot \mathrm{m}^{2}$ & $\theta_{\mathrm{O} 23}$ & $-63 \mathrm{~N}$ \\
$I_{23}$ & $0.02 \mathrm{~kg} \cdot \mathrm{m}^{2}$ & $\theta_{\mathrm{O} 33}$ & $-59 \mathrm{~N}$ \\
$I_{33}$ & $1.65 \mathrm{~kg} \cdot \mathrm{m}^{2}$ & & \\
\hline
\end{tabular}

The second step is the main procedure of the control experiment. When a desired curve is given, under the control of the adaptive robust control, the platform begins to follow the trajectory. During this process, online parameter estimation works to make the estimated unknown parameters subsequently closer to the real values as the algorithms are executed. Fig. 6 shows the tracking error performance and the position tracking in the joint space when given a desired sine curve on the roll axis in the working space without parameter initialization.
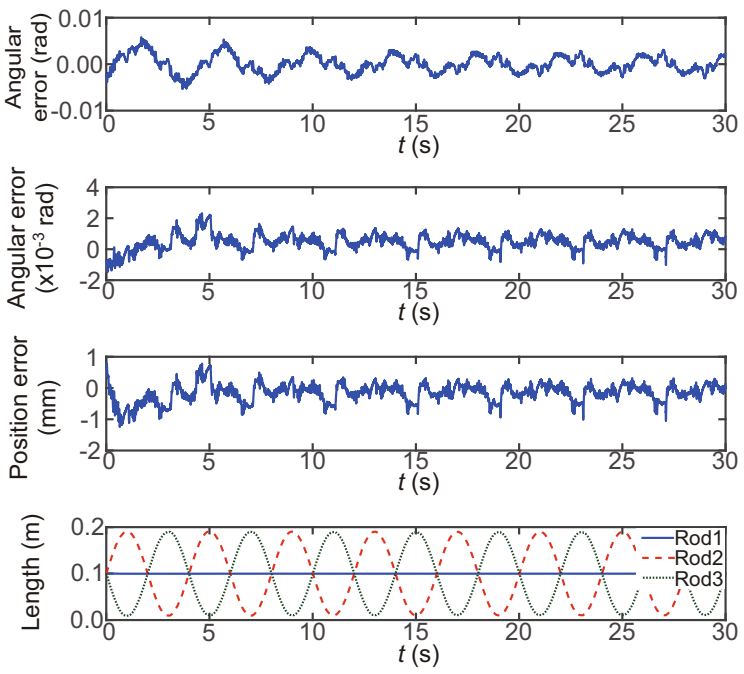

Fig. 6 Tracking without initialization $(m=20 \mathrm{~kg})$ : roll axis, pitch axis, and $Z$ axis tracking errors of 3-RPS, and length of each rod in the joint space
The last step is analyzing controller performance, which is used to make judgments about the effectiveness of different control algorithms and variable configurations. To quantify the performance, the data for the last $10 \mathrm{~s}$ is used to calculate the error indices. The indices include the maximum tracking error $e_{\mathrm{F}}$ and the root-mean-square error $\|e\|_{\mathrm{rms}}$, shown in Eq. (52). The relative error $\|e\|_{\mathrm{rms}} / A_{m}$ is calculated to represent the general effectiveness evaluation criteria of the controller, where $A_{m}$ is the amplitude of the trajectory.

$$
\left\{\begin{array}{l}
e_{\mathrm{F}}=\left.\max \{|e|\}\right|_{T_{\mathrm{f}}-10 \leq t \leq T_{\mathrm{f}}}, \\
\|e\|_{\mathrm{rms}}=\sqrt{\frac{1}{10} \int_{T_{\mathrm{f}}-10}^{T_{\mathrm{f}}} e^{2} \mathrm{~d} t} .
\end{array}\right.
$$

The experimental results shown in Figs. 6 and 7 demonstrate that if the initial value set was chosen properly, then the posture tracking should be good. Fig. 6 indicates the situation where a desired input trajectory is given in the working space without parameter initialization. When the initial parameters are all chosen not far from the true values, the performance is acceptable, because it is consistent with the simulation results. However, if the initial parameter settings are far from realistic, there will be some jitter introduced in the motion of the upper board. Moreover, as the model differences become larger, the platform may enter an unstable status. As shown in Fig. 7, the system works at the edge of stability and the tracking performance decreases.
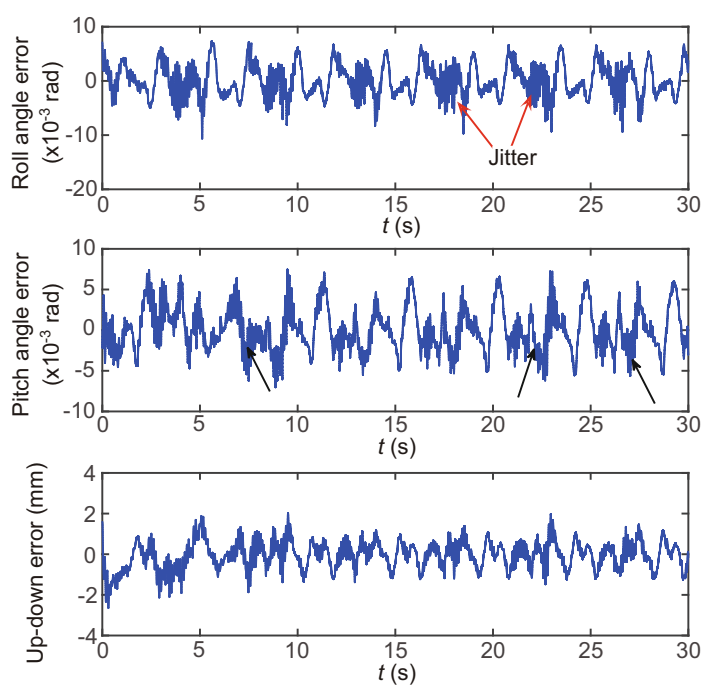

Fig. 7 Vibration caused by initial value difference and load change $(m=75 \mathrm{~kg})$ : roll angle, pitch angle, and up-down tracking errors of 3-RPS 
After applying the initialization step for a new load setup to the platform, it is quite common that the $4 \mathrm{D}$ movie chairs bear different audiences who have different weights and sitting positions, so the adverse effects can be eliminated and the jitter phenomenon will disappear (Fig. 8).


(a)
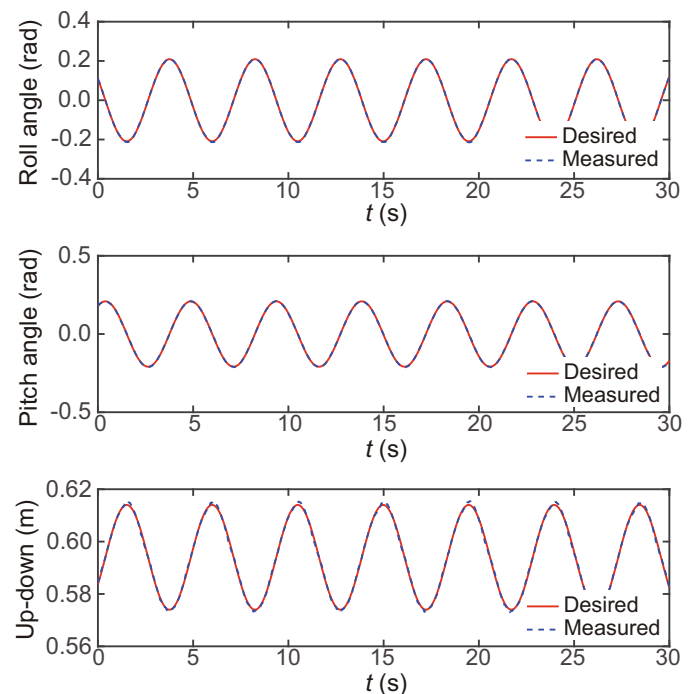

(b)

Fig. 8 Tracking with initialization $(m=75 \mathrm{~kg})$ : (a) roll angle, pitch angle, and up-down tracking errors of 3-RPS; (b) roll angle, pitch angle, and up-down tracking of $3-\mathrm{RPS}$

\section{Discussion and conclusions}

In this paper, an ARC controller with a parameter initialization method was proposed. It was found to be stable and effective while controlling the posture of a 3-RPS platform driven by pneumatic cylinders. The process of stability proof is shown in the Appendix.

Parameter uncertainty and nonlinear features can be estimated and inhibited by online estimation methods and robust parts of the controller. The experimental results indicated that the unknown parameters are indeed bounded and converge. The parameter identification not only prevents the vibration phenomenon but also decreases the estimation time when given a set of better initial parameter values. This represents important progress in practical settings, because the robustness of the system is improved when facing varying surroundings and external loads. Fig. 9 gives a comparison between the parameter estimation processes under ARC and ARCPI. After the initialization, the range of the estimated parameters is much smaller and more accurate. For a more complex situation, a compound
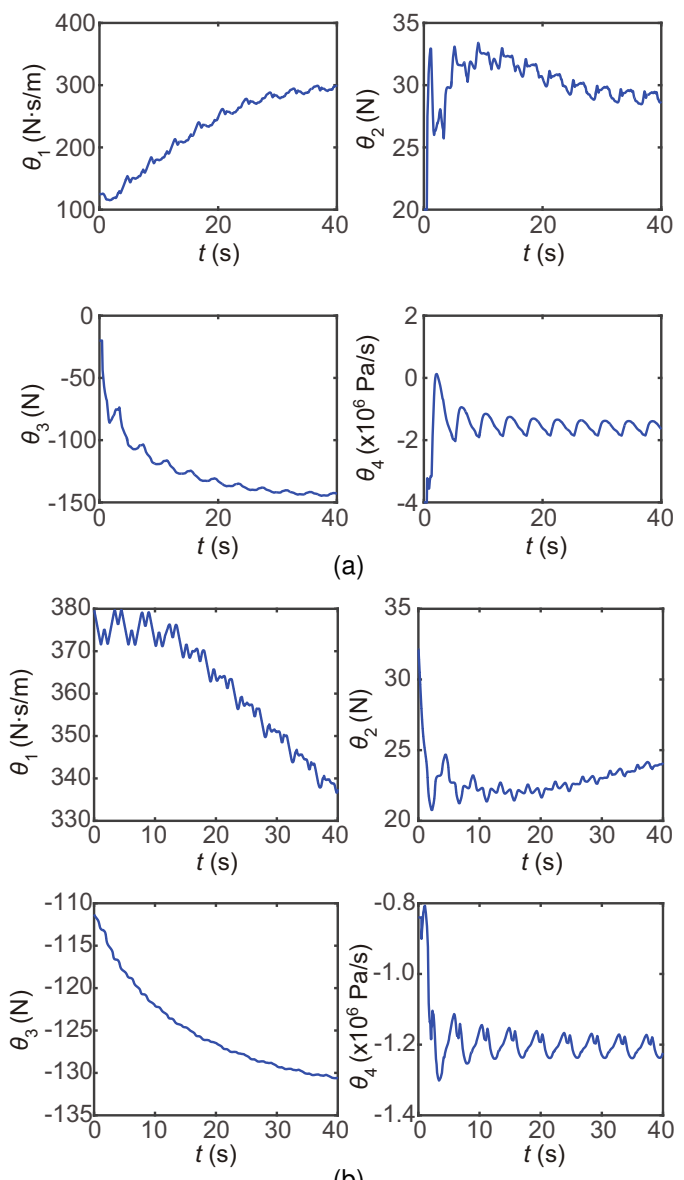

(b)

Fig. 9 Comparison of the parameter matrix without (ARC) (a) and with (ARCPI) (b) initialization 
sine wave $\boldsymbol{X}_{\mathrm{d}}$ is used as the desired curve in the working space. The posture tracking results are shown in Fig. 10 under ARCPI. Posture control experiments have also been performed using the same desired trajectory but different algorithms. The relative tracking error of ARCPI was not more than 1\%. Error analysis comparisons with several other algorithms are shown in Tables 3 and 4.
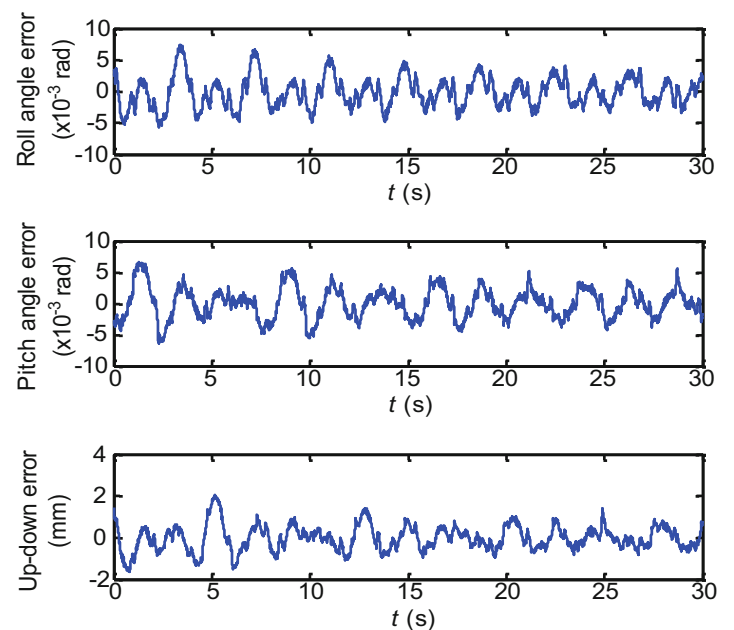

(a)


(b)

Fig. 10 Compound trajectory control performance: (a) roll angle, pitch angle, and up-down tracking errors of 3-RPS; (b) roll angle, pitch angle, and up-down tracking of 3-RPS

Compared to previous methods such as SMC and the basic ARC controller, the proposed method can improve the tracking performance by the integrated direct/indirect adaptive robust design.
Table 3 Tracking error analysis $e_{\mathrm{F}}(m=50 \mathrm{~kg})$

\begin{tabular}{lccc}
\hline Parameter & SMC & ARC & ARCPI \\
\hline Roll (rad) & 0.0110 & 0.0046 & 0.0032 \\
Roll & $3.9 \%$ & $1.6 \%$ & $1.1 \%$ \\
Pitch $(\mathrm{rad})$ & 0.0130 & 0.0057 & 0.0037 \\
Pitch $_{\text {rel }}$ & $4.1 \%$ & $1.8 \%$ & $1.2 \%$ \\
Heave $(\mathrm{mm})$ & 3.6 & 2.0 & 1.4 \\
Heave $_{\text {rel }}$ & $4.0 \%$ & $2.2 \%$ & $1.6 \%$ \\
\hline
\end{tabular}

Table 4 Tracking error analysis $e_{\mathrm{rms}}(m=50 \mathrm{~kg})$

\begin{tabular}{lccc}
\hline Parameter & SMC & ARC & ARCPI \\
\hline Roll (rad) & 0.0070 & 0.0023 & 0.0016 \\
Roll $_{\text {rel }}$ & $2.70 \%$ & $0.82 \%$ & $0.57 \%$ \\
Pitch $_{\text {rad })}$ & 0.0740 & 0.0028 & 0.0020 \\
Pitch $_{\text {rel }}$ & $2.30 \%$ & $0.88 \%$ & $0.63 \%$ \\
Heave $\left._{\text {mm }}\right)$ & 2.1 & 0.85 & 0.54 \\
Heave $_{\text {rel }}$ & $2.30 \%$ & $0.94 \%$ & $0.60 \%$ \\
\hline
\end{tabular}

Meanwhile, parameter initial value identification expands the controller's adaptability of different working statuses and satisfies realistic conditions.

There are still some difficulties to overcome. One is the valve's dead-zone features. The nonlinear dead-zone property is a major factor in control precision, but different valves have different dead-zone properties even for the same batch of valves. It is impossible to evaluate each one's dead-zone and central voltage $u_{\mathrm{dz}}$ and $u_{\mathrm{m}}$ before applying them to a 3-RPS platform. A solution must be proposed to improve the adaptability of the controller. Moreover, to achieve a higher precision for the posture, new friction models should be considered in the controller design, such as the LuGre model, which has been shown to be an effective model for the friction between the piston and cylinder wall (Khayati et al., 2009)

\section{References}

Andrievsky, B., Kazunin, D.V., Kostygova, D.M., et al., 2014. Control of pneumatically actuated 6-DOF Stewart platform for driving simulator. Proc. 19th Int. Conf. on Methods and Models in Automation and Robotics, p.663-668. http://dx.doi.org/10.1109/MMAR.2014.6957433

Atkeson, C.G., An, C.H., Hollerbach, J.M., 1985. Rigid body load identification for manipulators. Proc. 24th IEEE Conf. on Decision and Control, p.996-1002. http://dx.doi.org/10.1109/CDC.1985.268649

Carneiro, J.F., de Almeida, F.G., 2007. Heat transfer evaluation of industrial pneumatic cylinders. Proc. Instit. Mech. Eng. Part I: J. Syst. Contr. Eng., 221(1):119128. http://dx.doi.org/10.1243/09596518JSCE188 
Chen, Z., Yao, B., Wang, Q.F., 2013a. Accurate motion control of linear motors with adaptive robust compensation of nonlinear electromagnetic field effect. IEEE/ASME Trans. Mechatron., 18(3):1122-1129. http://dx.doi.org/10.1109/TMECH.2012.2197217

Chen, Z., Yao, B., Wang, Q.F., 2013b. Adaptive robust precision motion control of linear motors with integrated compensation of nonlinearities and bearing flexible modes. IEEE Trans. Ind. Inform., 9(2):965-973. http://dx.doi.org/10.1109/TII.2012.2225439

Chen, Z., Yao, B., Wang, Q.F., 2015. $\mu$-synthesis-based adaptive robust control of linear motor driven stages with high-frequency dynamics: a case study. IEEE/ASME Trans. Mechatron., 20(3):1482-1490. http://dx.doi.org/10.1109/TMECH.2014.2369454

Cheng, Y.M., Chen, Y.S., 2013. An angle trajectory tracking for a 3-DOF pneumatic motion platform by the NI compact RIO embedded system. J. Mech. Eng. Autom., 3:14-21.

Díaz-Rodríguez, M., Mata, V., Valera, Á., et al., 2010. A methodology for dynamic parameters identification of 3-DOF parallel robots in terms of relevant parameters. Mech. Mach. Theory, 45(9):1337-1356. http://dx.doi.org/10.1016/j.mechmachtheory.2010.04.007

Farhat, N., Mata, V., Page, Á., et al., 2008. Identification of dynamic parameters of a 3-DOF RPS parallel manipulator. Mech. Mach. Theory, 43(1):1-17. http://dx.doi.org/10.1016/j.mechmachtheory.2006.12.011

Girin, A., Plestan, F., Brun, X., et al., 2009. High-order sliding-mode controllers of an electropneumatic actuator: application to an aeronautic benchmark. IEEE Trans. Contr. Syst. Technol., 17(3):633-645. http://dx.doi.org/10.1109/TCST.2008.2002950

Goodwin, G.C., Mayne, D.Q., 1987. A parameter estimation perspective of continuous time model reference adaptive control. Automatica, 23(1):57-70.

http://dx.doi.org/10.1016/0005-1098(87)90118-X

Grewal, K.S., Dixon, R., Pearson, J., 2011. Control design for a pneumatically actuated parallel link manipulator. Proc. 21st Int. Conf. on Systems Engineering, p.43-48. http://dx.doi.org/10.1109/ICSEng.2011.16

Grewal, K.S., Dixon, R., Pearson, J., 2012. LQG controller design applied to a pneumatic Stewart-Gough platform. Int. J. Autom. Comput., 9(1):45-53. http://dx.doi.org/10.1007/s11633-012-0615-7

Grotjahn, M., Heimann, B., Abdellatif, H., 2004. Identification of friction and rigid-body dynamics of parallel kinematic structures for model-based control. Multibody Syst. Dynam., 11(3):273-294. http://dx.doi.org/10.1023/B:MUBO.0000029426.05860. c2

Khayati, K., Bigras, P., Dessaint, L.A., 2009. LuGre modelbased friction compensation and positioning control for a pneumatic actuator using multi-objective outputfeedback control via LMI optimization. Mechatronics, 19(4):535-547. http://dx.doi.org/10.1016/j.mechatronics.2008.12.006

Kimura, T., Hara, S., Fujita, T., et al., 1997. Feedback linearization for pneumatic actuator systems with static friction. Contr. Eng. Pract., 5(10):1385-1394. http://dx.doi.org/10.1016/S0967-0661(97)00135-4

Meng, D., Tao, G., Chen, J., et al., 2011. Modeling of a pneumatic system for high-accuracy position control. Proc.
Int. Conf. on Fluid Power and Mechatronics, p.505-510. http://dx.doi.org/10.1109/FPM.2011.6045817

Meng, D., Tao, G., Zhu, X., 2013. Integrated direct/indirect adaptive robust motion trajectory tracking control of pneumatic cylinders. Int. J. Contr., 86(9):1620-1633. http://dx.doi.org/10.1080/00207179.2013.792002

Merlet, J.P., 2002. Parallel Robots. Kluwer Academic Publishers, Norwell, MA, USA.

Pfreundschuh, G.H., Kumar, V., Sugar, T.G., 1991. Design and control of a 3-DOF in-parallel actuated manipulator. IEEE Int. Conf. on Robotics and Automation, p.1659-1664

http://dx.doi.org/10.1109/ROBOT.1991.131857

Pradipta, J., Klünder, M., Weickgenannt, M., et al., 2013. Development of a pneumatically driven flight simulator Stewart platform using motion and force control. Proc. IEEE/ASME Int. Conf. on Advanced Intelligent Mechatronics, p.158-163. http://dx.doi.org/10.1109/AIM.2013.6584085

Ramsauer, M., Kastner, M., Ferrara, P., et al., 2012. A pneumatically driven Stewart platform used as fault detection device. Appl. Mech. Mater., 186:227-233. http://dx.doi.org/10.4028/www.scientific.net/AMM.186. 227

Richardson, R., Plummer, A.R., Brown, M.D., 2001. Selftuning control of a low-friction pneumatic actuator under the influence of gravity. IEEE Trans. Contr. Syst. Technol., 9(2):330-334. http://dx.doi.org/10.1109/87.911384

Schulte, H., Hahn, H., 2004. Fuzzy state feedback gain scheduling control of servo-pneumatic actuators. Contr. Eng. Pract., 12(5):639-650. http://dx.doi.org/10.1016/S0967-0661(03)00148-5

Smaoui, M., Brun, X., Thomasset, D., 2006. A study on tracking position control of an electropneumatic system using backstepping design. Contr. Eng. Pract., 14(8):923-933. http://dx.doi.org/10.1016/j.conengprac.2005.05.003

Tao, G., Zuo, H., 2014. Cross-coupling adaptive robust control study of single/multiple 3-DOF pneumatic parallel platforms. Proc. 9th JFPS Int. Symp. on Fluid Power.

Wang, J., Fan, L., Hu, L., 2005. Positional forward solution and numeric-symbolic solution of singular configuration analysis for 3-RPS parallel platform mechanism. J. Mach. Des., 22(5):15-19 (in Chinese).

Yao, B., Tomizuka, M., 1997. Adaptive robust control of SISO nonlinear systems in a semi-strict feedback form. Automatica, 33(5):893-900. http://dx.doi.org/10.1016/S0005-1098(96)00222-1

Yao, J., Jiao, Z., Ma, D., 2014a. Extended-state-observerbased output feedback nonlinear robust control of hydraulic systems with backstepping. IEEE Trans. Ind. Electron., 61(11):6285-6293. http://dx.doi.org/10.1109/TIE.2014.2304912

Yao, J., Jiao, Z., Ma, D., et al., 2014b. High-accuracy tracking control of hydraulic rotary actuators with modeling uncertainties. IEEE/ASME Trans. Mechatron., $\mathbf{1 9}(2)$ :633-641. http://dx.doi.org/10.1109/TMECH.2013.2252360

Zheng, K.J., Cui, P., Guo, H.J., 2011. Kinematics and static characteristics analysis of 3-RPS parallel mechanism. J. Mach. Des., 28(9) (in Chinese). 
Zhu, X., Tao, G., Yao, B., et al., 2008. Adaptive robust posture control of a parallel manipulator driven by pneumatic muscles. Automatica, 44(9):2248-2257. http://dx.doi.org/10.1016/j.automatica.2008.01.015

Zhu, X., Tao, G., Yao, B., et al., 2009. Integrated direct/indirect adaptive robust posture trajectory tracking control of a parallel manipulator driven by pneumatic muscles. IEEE Trans. Contr. Syst. Technol., $\mathbf{1 7}(3)$ :576-588.

http://dx.doi.org/10.1109/TCST.2008.2001715

\section{Appendix: Proof of stability}

According to the system dynamic model and definitions of $\boldsymbol{e}_{\mathrm{s}}$, define a Lyapunov function:

$$
V_{1}=\frac{1}{2} e_{\mathrm{s}}^{\mathrm{T}} \boldsymbol{M}_{\mathrm{e}} \boldsymbol{e}_{\mathrm{s}}
$$

With Eqs. (11) and (34), the derivation of $V_{1}$ is

$$
\dot{V}_{1}=\boldsymbol{e}_{\mathrm{s}}^{\mathrm{T}}\left(\bar{A}_{a} \boldsymbol{p}_{\mathrm{L}}+\boldsymbol{\varphi}_{a}^{\mathrm{T}} \boldsymbol{\theta}_{a}+\tilde{\boldsymbol{f}}_{0}-\boldsymbol{M}_{\mathrm{e}} \ddot{\boldsymbol{x}}_{\mathrm{d}}+\boldsymbol{M}_{\mathrm{e}} \boldsymbol{K}_{\boldsymbol{x}} \boldsymbol{e}\right) \text {. }
$$

Substituting Eqs. (35) and (36) into Eq. (A2) yields

$$
\begin{aligned}
\dot{V}_{1}= & \bar{A}_{a} \boldsymbol{e}_{\mathrm{s}}^{\mathrm{T}} \boldsymbol{e}_{\mathrm{p}}-\boldsymbol{e}_{\mathrm{s}}^{\mathrm{T}} \boldsymbol{K}_{\mathrm{p}} \boldsymbol{e}_{\mathrm{s}} \\
& +\boldsymbol{e}_{\mathrm{s}}^{\mathrm{T}}\left(\bar{A}_{a} \boldsymbol{p}_{\mathrm{Ld} 22}+\bar{A}_{a} \boldsymbol{p}_{\mathrm{Lds} 2}-\boldsymbol{\varphi}_{a}^{\mathrm{T}} \tilde{\boldsymbol{\theta}}_{a}+\tilde{\boldsymbol{f}}_{0}\right) .
\end{aligned}
$$

According to the definition of $\boldsymbol{p}_{\mathrm{Ld}}$, we have

$$
\begin{aligned}
\dot{V}_{2}= & \bar{A}_{a} \boldsymbol{e}_{\mathrm{s}}^{\mathrm{T}} \boldsymbol{e}_{\mathrm{p}}-\boldsymbol{e}_{\mathrm{s}}^{\mathrm{T}} \boldsymbol{K}_{\mathrm{p}} \boldsymbol{e}_{\mathrm{s}} \\
& +\boldsymbol{e}_{\mathrm{s}}^{\mathrm{T}}\left(\bar{A}_{a} \boldsymbol{p}_{\mathrm{Lds} 2}-\tilde{\boldsymbol{d}}_{\mathrm{c} 1}+\boldsymbol{R}_{1}(t)\right) .
\end{aligned}
$$

According to Theorem 2 and Eq. (39), $\boldsymbol{p}_{\mathrm{Lds} 2}$ satisfies

$$
\left\{\begin{array}{l}
\boldsymbol{e}_{\mathrm{s}}^{\mathrm{T}}\left(\bar{A}_{a} \boldsymbol{p}_{\mathrm{Lds} 2}-\tilde{\boldsymbol{d}}_{\mathrm{c} 1}+\boldsymbol{R}_{1}(t)\right) \leq \eta_{1}, \\
\bar{A}_{a} \boldsymbol{e}_{\mathrm{s}}^{\mathrm{T}} \boldsymbol{p}_{\mathrm{Lds} 2} \leq 0
\end{array}\right.
$$

Then, the derivation of $V_{1}$ can be rewritten in the form

$$
\dot{V}_{1} \leq \bar{A}_{a} \boldsymbol{e}_{\mathrm{s}}^{\mathrm{T}} \boldsymbol{e}_{\mathrm{p}}-\boldsymbol{e}_{\mathrm{s}}^{\mathrm{T}} \boldsymbol{K}_{\mathrm{p}} \boldsymbol{e}_{\mathrm{s}}+\eta_{1} .
$$

Define another Lyapunov function $V_{2}$ :

$$
V_{2}=V_{1}+\frac{1}{2} e_{\mathrm{p}}^{\mathrm{T}} e_{\mathrm{p}}
$$

As in the previous step, derive $V_{2}$ :

$$
\begin{aligned}
\dot{V}_{2}= & \left.\dot{V}_{1}\right|_{p_{\mathrm{Ld}}}-\boldsymbol{e}_{\mathrm{p}}^{\mathrm{T}} \boldsymbol{K}_{\mathrm{q}} \boldsymbol{e}_{\mathrm{p}} \\
& +\boldsymbol{e}_{\mathrm{p}}^{\mathrm{T}}\left(\boldsymbol{q}_{\mathrm{Ld} 22}+\boldsymbol{q}_{\mathrm{Lds} 2}-\boldsymbol{\varphi}_{b}^{\mathrm{T}} \tilde{\boldsymbol{\theta}}_{b}+\tilde{\boldsymbol{d}}_{0}\right) .
\end{aligned}
$$

Based on the definition of $\boldsymbol{q}_{\mathrm{Ld}}, \dot{V}_{2}$ is expressed as

$$
\begin{aligned}
\dot{V}_{2}= & \left.\dot{V}_{1}\right|_{p_{\mathrm{Ld}}}-\boldsymbol{e}_{\mathrm{p}}^{\mathrm{T}} \boldsymbol{K}_{\mathrm{q}} \boldsymbol{e}_{\mathrm{p}} \\
& +\boldsymbol{e}_{\mathrm{p}}^{\mathrm{T}}\left(\boldsymbol{q}_{\mathrm{Lds} 2}-\tilde{\boldsymbol{d}}_{\mathrm{c} 2}+\boldsymbol{R}_{2}(t)\right) .
\end{aligned}
$$

According to Theorem 3 and Eq. (45), $\boldsymbol{q}_{\mathrm{Lds} 2}$ satisfies

$$
\left\{\begin{array}{l}
\boldsymbol{e}_{\mathrm{p}}^{\mathrm{T}}\left(\boldsymbol{q}_{\mathrm{Lds} 2}-\tilde{\boldsymbol{d}}_{\mathrm{c} 2}+\boldsymbol{R}_{2}(t)\right) \leq \eta_{2}, \\
\boldsymbol{e}_{\mathrm{p}}^{\mathrm{T}} \boldsymbol{q}_{\mathrm{Lds} 2} \leq 0,
\end{array}\right.
$$

which means

$$
\dot{V}_{2} \leq-\boldsymbol{e}_{\mathrm{s}}^{\mathrm{T}} \boldsymbol{K}_{\mathrm{p}} \boldsymbol{e}_{\mathrm{s}}-\boldsymbol{e}_{\mathrm{p}}^{\mathrm{T}} \boldsymbol{K}_{\mathrm{q}} \boldsymbol{e}_{\mathrm{p}}+\eta_{1}+\eta_{2} .
$$

According to Lyapunov theory, $\dot{V}_{2}$ gradually converges to a spherical area, and the size is controlled by the values of $\boldsymbol{K}_{\mathrm{p}}, \boldsymbol{K}_{\mathrm{q}}, \eta_{1}, \eta_{2}$, which means the tracking error vector $\boldsymbol{e}$ is bounded. Thus, the controller designed in Section 3.3 is stable. 